\title{
Comparative functional pan-genome analyses to build connections between genomic dynamics and phenotypic evolution in polycyclic aromatic hydrocarbon metabolism in the genus Mycobacterium
}

Ohgew Kweon ${ }^{1 \dagger}$, Seong-Jae Kim ${ }^{1 \dagger}$, Jochen Blom², Sung-Kwan Kim³ ${ }^{3}$ Bong-Soo Kim², Dong-Heon Baek ${ }^{5}$, Su Inn Park ${ }^{6}$, John B Sutherland ${ }^{1}$ and Carl E Cerniglia ${ }^{*}$

\begin{abstract}
Background: The bacterial genus Mycobacterium is of great interest in the medical and biotechnological fields. Despite a flood of genome sequencing and functional genomics data, significant gaps in knowledge between genome and phenome seriously hinder efforts toward the treatment of mycobacterial diseases and practical biotechnological applications. In this study, we propose the use of systematic, comparative functional pan-genomic analysis to build connections between genomic dynamics and phenotypic evolution in polycyclic aromatic hydrocarbon (PAH) metabolism in the genus Mycobacterium.

Results: Phylogenetic, phenotypic, and genomic information for 27 completely genome-sequenced mycobacteria was systematically integrated to reconstruct a mycobacterial phenotype network (MPN) with a pan-genomic concept at a network level. In the MPN, mycobacterial phenotypes show typical scale-free relationships. PAH degradation is an isolated phenotype with the lowest connection degree, consistent with phylogenetic and environmental isolation of PAH degraders. A series of functional pan-genomic analyses provide conserved and unique types of genomic evidence for strong epistatic and pleiotropic impacts on evolutionary trajectories of the PAH-degrading phenotype. Under strong natural selection, the detailed gene gain/loss patterns from horizontal gene transfer (HGT)/deletion events hypothesize a plausible evolutionary path, an epistasis-based birth and pleiotropy-dependent death, for PAH metabolism in the genus Mycobacterium. This study generated a practical mycobacterial compendium of phenotypic and genomic changes, focusing on the PAH-degrading phenotype, with a pan-genomic perspective of the evolutionary events and the environmental challenges.

Conclusions: Our findings suggest that when selection acts on PAH metabolism, only a small fraction of possible trajectories is likely to be observed, owing mainly to a combination of the ambiguous phenotypic effects of PAHs and the corresponding pleiotropy- and epistasis-dependent evolutionary adaptation. Evolutionary constraints on the selection of trajectories, like those seen in PAH-degrading phenotypes, are likely to apply to the evolution of other phenotypes in the genus Mycobacterium.
\end{abstract}

Keywords: Mycobacterium, PAH metabolism, Pan-genome, Functional genomics, Functional pan-genome, Phenotype network, Evolution, Epistasis, Pleiotropy

\footnotetext{
* Correspondence: carl.cerniglia@fda.hhs.gov

${ }^{\dagger}$ Equal contributors

${ }^{1}$ Division of Microbiology, National Center for Toxicological Research/FDA

Jefferson, Arkansas, USA

Full list of author information is available at the end of the article
}

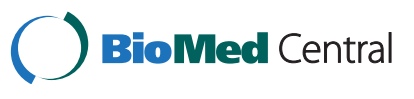

(c) 2015 Kweon et al.; licensee BioMed Central. This is an Open Access article distributed under the terms of the Creative Commons Attribution License (http://creativecommons.org/licenses/by/4.0), which permits unrestricted use, distribution, and reproduction in any medium, provided the original work is properly credited. The Creative Commons Public Domain Dedication waiver (http://creativecommons.org/publicdomain/zero/1.0/) applies to the data made available in this article, unless otherwise stated. 


\section{Background}

Mycobacterium is the single genus in the family Mycobacteriaceae, in the order Actinomycetales [1]. There are more than 150 recognized parasitic and free-living species of Mycobacterium (http://www.bacterio.cict.fr/m/mycobacterium.html) with remarkable metabolic and physiologic versatility, enabling colonization of diverse habitats [2,3]. There has been great interest in mycobacteria due to their abilities both to cause devastating diseases in humans and animals and to degrade toxic compounds in the environment $[4,5]$. For these reasons, the number of available genome sequences has been growing fast and genome-scale omics data have been generated, allowing deeper functional genomics insights into their phenotypic features [6-13]. Several mycobacterial models have played an important role in the functional genomics understanding of basic phenotypic traits. For example, M. tuberculosis and M. leprae, for mycobacterial pathogenicity, and M. vanbaalenii PYR-1, for high-molecular-weight (HMW) PAH biodegradation, have been studied and the accumulated information has been applied to obtain information on other species $[3,5,14,15]$. However, despite the exciting discoveries continually adding to the broader and deeper knowledge of mycobacteria, there is a significant knowledge gap between genome and phenome in the genus Mycobacterium, due mainly to the epistatic and pleiotropic complexity of the genomic and phenotypic traits. The epistatic interaction-functional combination of two or more genes (or enzymes) for a single trait (or phenotype)-and pleiotropic activity-functional contribution of a gene (or enzyme) for multiple traits (or phenotypes) - are still poorly understood at the genome level [16] but are essential for a deeper understanding of mycobacterial systems.

Newly arising genomic variations create phenotypic changes upon which natural selection can act. The evolutionary genetics of epistasis and pleiotropy not only make their effects on evolution difficult to predict, but also constrain the path of natural selection, which allows observation of only a small fraction of possible evolutionary trajectories [16]. In this respect, mycobacterial HMW $\mathrm{PAH}$ degradation is of particular interest, because of the metabolic ambiguity (as nutrient or toxicant) (Figure 1) and the environment-dependent availability of HMW PAHs [8]. As shown in Figure 1, PAHs serve not only as nutrients to be metabolized but also as potential toxicants if they are transformed to diol-epoxides by metabolic activation [17-19]. PAHs consist of two or more fused aromatic rings arranged in various configurations [20].

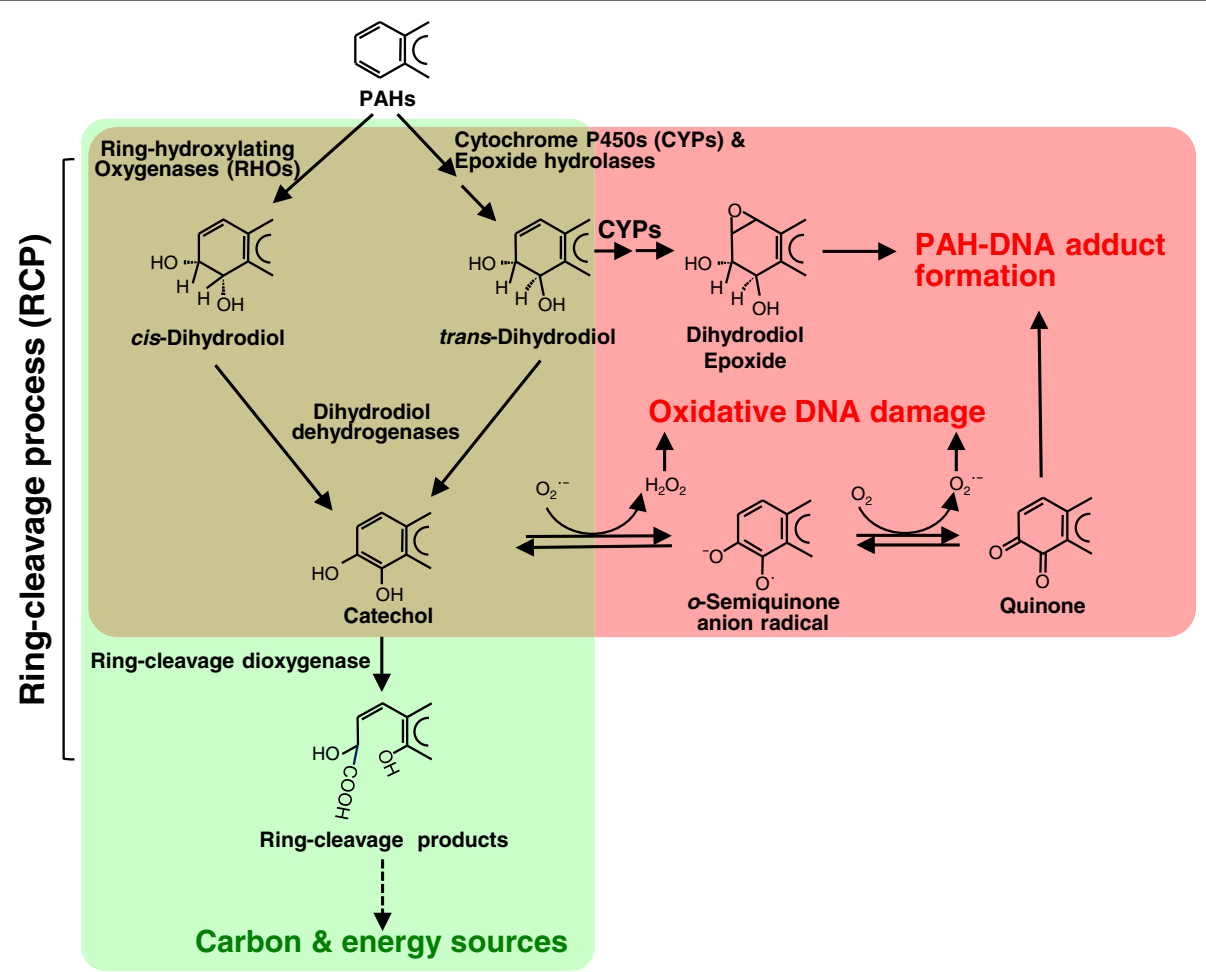

Figure 1 Metabolic ambiguity of PAHs as nutrient or toxicant. The ring-cleavage process (RCP)—-mono- or dioxygenation by ring-hydroxylating oxygenases (RHOs) or cytochrome P450 monooxygenases (CYPs), dehydrogenation by dihydrodiol dehydrogenase, and ring-cleavage by ring-cleavage dioxygenase - in mycobacterial PAH metabolism responsible for metabolic benefits is in the green colored area, while production of toxic PAH metabolites/byproducts and reactive oxygen species (ROS), which have the potential to cause PAH-adducts and oxidative DNA damage, is in the red colored area. 
Although PAHs are introduced into the environment mainly from anthropogenic routes, they are also found occurring naturally in the environment. Since physical and chemical characteristics of PAHs vary with molecular weight, PAHs can be divided into two groups, lowmolecular-weight PAHs (LMW PAHs), with two or three fused aromatic rings, and HMW PAHs, with four or more fused aromatic rings. HMW PAHs are more toxic and difficult to degrade, persisting longer than LMW PAHs in the environment [20]. Microbial metabolic activity represents one of the primary processes of degradation and transformation of PAHs in the environment [20], and some members of the genus Mycobacterium have been shown to be able to degrade HMW PAHs $[12,21,22]$. Therefore, a possible full evolutionary scenario for PAH metabolism in the genus Mycobacterium could provide deeper insights into the relationship between genomic variations and natural selection.

M. vanbaalenii PYR-1 has been extensively studied as a model microorganism for bacterial HMW PAH degradation at both laboratory and field scales $[5,14,15]$. Recently, a PAH metabolic network (PAH-MN), including HMW PAHs, has been systematically reconstructed using genomic, proteomic, and metabolic information [9]. According to the PAH-MN, PAH substrates are degraded by a set of interconnected functional processes, termed ringcleavage processes (RCPs), side-chain processes (SCPs), and central aromatic processes (CAPs). Under the common aromatic metabolic logic (the activation of benzene rings and ring opening), the oxygen-dependent activation of thermodynamically stable benzene rings and ring cleavage occurs in RCP, the side chain processing of the opened ring to produce biological metabolic precursors occurs in SCP, and the metabolic connection of protocatechuate to the tricarboxylic acid (TCA) cycle occurs in CAP. In our previous study with $M$. vanbaalenii PYR-1, a fundamental building principle of the PAH-MN, a patchwork assembly of the phylogenetic modules, with a backward evolutionary direction from CAP via SCP to RCP, has been hypothesized on the basis of the functional association of the phylogenetic modules [9]. When considering the ambiguous metabolic effects of HMW PAHs, the model microorganism can benefit from the buildup of the PAH-MN in both architectural and functional aspects. That is, the directed growth of the metabolic network from CAP via SCP to RCP increases the architectural and functional connectivity among phylogenetic modules with $\mathrm{PAH}-$ degrading enzymes, resulting in decreasing the epimetabolome, including toxic intermediates, such as diol epoxides [9]. Therefore, the backward evolutionary growth of the PAH-MN can also have a practical toxicological benefit that minimizes toxic effects of the activated PAH intermediates, which are produced in RCP. As revealed in the proteomic analysis of the PAH-MN, function-specific regulation of the functional modules (RCP, SCP, and CAP) completes the evolutionary and metabolic endeavors for nutritional benefits from PAHs, by allowing mature metabolic behavior of the channel management for global PAH metabolism with more productivity and less toxicity (that is, generating more productive biological precursors, such as pyruvate and acetyl-CoA, and fewer toxic intermediates, such as $o$-quinones) [9]. Therefore, it is evident that mycobacterial $\mathrm{PAH}$ metabolism is an epistasis-centric phenotype, which is essential for functional combination of two or more genetic modules and requires active investment to obtain and continuous, sophisticated management to keep. However, no pan-genomic evidence has been evaluated for this hypothesis, and consequently, a full evolutionary story of the PAH-degrading phenotype in the genus Mycobacterium has not been proposed.

Phenotypic traits of living cells are the result of successful functional interactions among biochemical compounds in highly complex biochemical networks [9,23]. It is a major challenge to understand causes and consequences of evolution of the phenotypes. In this study, to retrace plausible evolutionary trajectories of $\mathrm{PAH}$ metabolism in the genus Mycobacterium with no anthropomorphic bias, we employed three key concepts: networks, pan-genomic comparison, and functional genomics. First, networks allow for intuitive platforms for connection and visualization of the interacting parts with a more holistic view of phenotypic interactions in the MPN and functional interactions of the genetic modules in the PAH-MN. Second, pan-genomic comparison of the mycobacterial genomes, which exhibit extreme levels of evolutionary plasticity with high levels of gene gain and loss during evolution [24], allows integrating the genome sources to increase the power of genomic analyses. Finally, functional genomic data (proteomic data in this study) filter out the static genes that are not expressed and so may not be involved in a given phenotype from the pan-genomic data to provide phenotype-related functional pan-genomic dynamics. This multidisciplinary approach, which requires intensive systematic integration of the three different types of data, was essential to elucidate the nature and underlying mechanisms, and the phenotypic feedbacks, of epistatic and pleiotropic evolutionary effects of PAH metabolism in the genus Mycobacterium.

\section{Results and discussion}

\section{Systematic integration of data}

In this study, initially, phylogeny, phenotypes, and genomic contents of 27 completely genome-sequenced mycobacteria were systematically integrated to generate an applicable mycobacterial compendium of phenotypic and genomic properties (Table 1). On the basis of the integrated information, an MPN was reconstructed and analyzed from a pan-genomic perspective. A series of consecutive functional pan-genomic analyses provided conserved and unique 
Table 1 Phenotypic and genomic properties of the 27 completely genome-sequenced strains of mycobacteria

\begin{tabular}{|c|c|c|c|c|c|c|c|c|c|}
\hline \multirow[t]{2}{*}{ Mycobacterial strain } & \multicolumn{4}{|c|}{ Phenotypic properties } & \multicolumn{4}{|c|}{ Genomic properties } & \multirow[t]{2}{*}{ Reference } \\
\hline & Life-style $^{a}$ & $\begin{array}{l}\text { Growth } \\
\text { rate }\end{array}$ & Pathogenicity & PAH-metabolism & $\begin{array}{l}\text { Genome } \\
\text { size }\end{array}$ & GC \% & $\begin{array}{l}\text { Gene } \\
\text { count }\end{array}$ & $\begin{array}{l}\text { COG } \\
\text { count }\end{array}$ & \\
\hline Mycobacterium smegmatis MC $^{2} 155$ & $\mathrm{FL}$ & Fast & Non-pathogenic & PAH-non-degrading & 6988209 & 0.67 & 6941 & 4796 & {$[25]$} \\
\hline Mycobacterium vanbaalenii PYR-1 & $\mathrm{FL}$ & Fast & Non-pathogenic & PAH-degrading & 6491865 & 0.68 & 6047 & 4186 & {$[26,27]$} \\
\hline Mycobacterium sp. KMS & $\mathrm{FL}$ & Fast & Non-pathogenic & PAH-degrading & 6256079 & 0.68 & 6089 & 4135 & {$[12]$} \\
\hline Mycobacterium sp. JLS & $\mathrm{FL}$ & Fast & Non-pathogenic & PAH-degrading & 6048425 & 0.68 & 5855 & 4148 & {$[12]$} \\
\hline Mycobacterium gilvum PYR-GCK & $\mathrm{FL}$ & Fast & Non-pathogenic & PAH-degrading & 5982829 & 0.68 & 5683 & 3901 & {$[28]$} \\
\hline Mycobacterium sp. MCS & $\mathrm{FL}$ & Fast & Non-pathogenic & PAH-degrading & 5920523 & 0.68 & 5704 & 3955 & {$[12,29]$} \\
\hline Mycobacterium gilvum Spyr1 & $\mathrm{FL}$ & Fast & Non-pathogenic & PAH-degrading & 5783292 & 0.68 & 5434 & 4038 & {$[13]$} \\
\hline Mycobacterium sp. JDM601 & $\mathrm{FHA}$ & Slow & Pathogenic & PAH-non-degrading & 4643668 & 0.68 & 4398 & 3162 & {$[30]$} \\
\hline $\begin{array}{l}\text { Mycobacterium marinum M, } \\
\text { ATCC BAA-535 }\end{array}$ & FHA & Slow & Pathogenic & PAH-non-degrading & 6660144 & 0.66 & 5501 & 3717 & {$[31]$} \\
\hline Mycobacterium ulcerans Agy99 & $\mathrm{FHA}$ & Slow & Pathogenic & PAH-non-degrading & 5805761 & 0.65 & 4306 & 2853 & {$[32,33]$} \\
\hline Mycobacterium avium 104 & FHA & Slow & Pathogenic & PAH-non-degrading & 5475491 & 0.69 & 5305 & 3504 & {$[34,35]$} \\
\hline $\begin{array}{l}\text { Mycobacterium abscessus CIP } \\
104536\end{array}$ & FHA & Fast & Pathogenic & PAH-non-degrading & 5090491 & 0.64 & 4991 & 3301 & {$[36]$} \\
\hline $\begin{array}{l}\text { Mycobacterium avium subsp. } \\
\text { paratuberculosis K-10 }\end{array}$ & FHA & Slow & Pathogenic & PAH-non-degrading & 4829781 & 0.69 & 4415 & 3188 & {$[37-39]$} \\
\hline $\begin{array}{l}\text { Mycobacterium canettii CIPT } \\
140010059\end{array}$ & $\mathrm{FHA}$ & Slow & Pathogenic & PAH-non-degrading & 4482059 & 0.66 & 3909 & 2949 & {$[40]$} \\
\hline $\begin{array}{l}\text { Mycobacterium tuberculosis F11 } \\
\text { (ExPEC) }\end{array}$ & FHA & Slow & Pathogenic & PAH-non-degrading & 4424435 & 0.66 & 4019 & 2791 & {$[41]$} \\
\hline Mycobacterium tuberculosis H37Ra & FHA & Slow & Pathogenic & PAH-non-degrading & 4419977 & 0.66 & 4099 & 2811 & {$[42-44]$} \\
\hline $\begin{array}{l}\text { Mycobacterium tuberculosis H37Rv } \\
\text { (lab strain) }\end{array}$ & $\mathrm{FHA}$ & Slow & Pathogenic & PAH-non-degrading & 4411532 & 0.66 & 4062 & 2807 & {$[44]$} \\
\hline Mycobacterium tuberculosis CCDC5180 & FHA & Slow & Pathogenic & PAH-non-degrading & 4405981 & 0.66 & 3639 & 2836 & {$[40,45,46]$} \\
\hline Mycobacterium tuberculosis CDC1551 & FHA & Slow & Pathogenic & PAH-non-degrading & 4403706 & 0.66 & 4300 & 2716 & {$[47,48]$} \\
\hline Mycobacterium tuberculosis CCDC5079 & FHA & Slow & Pathogenic & PAH-non-degrading & 4398812 & 0.66 & 3695 & 2875 & {$[49]$} \\
\hline $\begin{array}{l}\text { Mycobacterium tuberculosis KZN } \\
1435 \text { (MDR) }\end{array}$ & $\mathrm{FHA}$ & Slow & Pathogenic & PAH-non-degrading & 4398250 & 0.66 & 4107 & 2799 & {$[25,29]$} \\
\hline Mycobacterium africanum GM041182 & FHA & Slow & Pathogenic & PAH-non-degrading & 4389314 & 0.66 & 3880 & 2896 & {$[50,51]$} \\
\hline $\begin{array}{l}\text { Mycobacterium bovis BCG Pasteur } \\
1173 \mathrm{P} 2\end{array}$ & FHA & Slow & Pathogenic & PAH-non-degrading & 4374522 & 0.66 & 4048 & 2783 & {$[42,43]$} \\
\hline Mycobacterium bovis BCG Tokyo 172 & FHA & Slow & Pathogenic & PAH-non-degrading & 4371711 & 0.66 & 3996 & 2776 & {$[52]$} \\
\hline Mycobacterium bovis AF2122/97 & FHA & Slow & Pathogenic & PAH-non-degrading & 4345492 & 0.66 & 4014 & 2760 & {$[53]$} \\
\hline Mycobacterium leprae TN & Ol & Slow & Pathogenic & PAH-non-degrading & 3268203 & 0.58 & 2750 & 1175 & {$[53-55]$} \\
\hline Mycobacterium leprae Br4923 & $\mathrm{Ol}$ & Slow & Pathogenic & PAH-non-degrading & 3268071 & 0.58 & 1654 & 1173 & {$[56]$} \\
\hline
\end{tabular}

${ }^{\mathrm{a}} \mathrm{FL}$, free-living; FHA, facultatively-host-associated; OI, obligately intracellular.

genomic evidence for strong epistatic and pleiotropic impacts on the evolutionary trajectories of the PAHdegrading phenotypes in the genus Mycobacterium.

A compendium of phenotypic and genomic changes on the bases of phylogeny, genomic contents, and phenotypes in the genus Mycobacterium

In this study, several types of information, including phylogenetic, genomic and phenotypic traits, were integrated to offer a rational comparative clustering of the 27 mycobacterial strains (Table 1), which allowed the following systematic pangenomic analyses of the evolutionary events in terms of mycobacterial PAH metabolism. Figure 2 shows comprehensive relationships of the 27 mycobacterial strains with respect to phylogeny, genome, and phenotypic traits.

\section{Phenotypic dichotomy of the well-characterized 27 mycobacterial strains}

Classical methods to catalogue mycobacteria are based on convenient phenotypic traits, such as growth rate, 


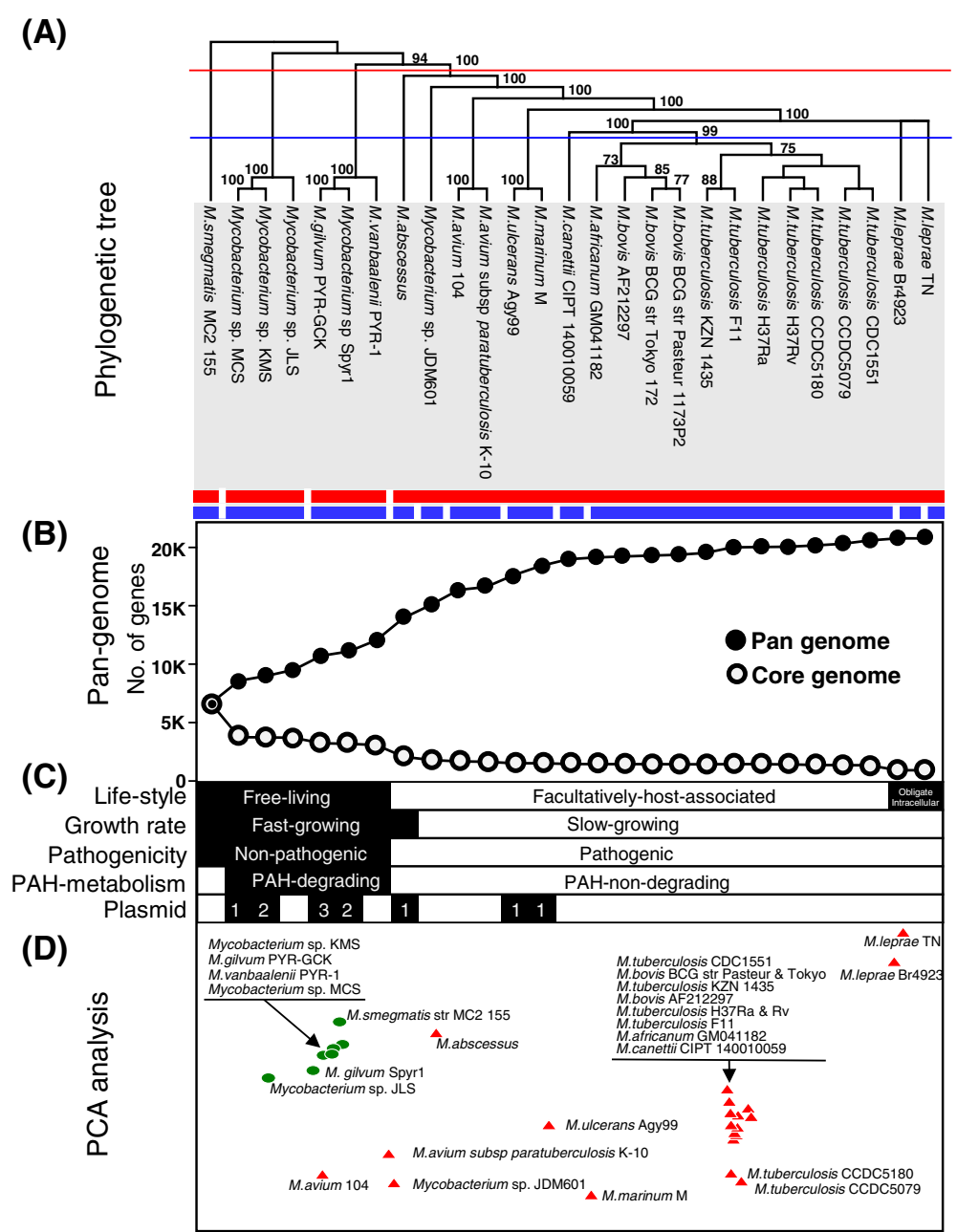

Figure 2 Clustering and pan-genomic change of the $\mathbf{2 7}$ fully genome-sequenced mycobacteria. (A) Maximum likelihood tree was constructed by concatenation of the 22 conserved genes in the 27 mycobacteria (available on TreeBASE S16971). The tree was rooted using the free-living M. smegmatis $M^{2} C^{2}$ 155, with the largest genome size, as an outgroup. The branches were annotated with bootstrap support. (B) Effect on the pan- and core genome dynamics found by a progressive addition of genomes based on phylogeny. (C) Dichotomous phenotype clustering corresponding to phylogeny for lifestyle, growth rate, pathogenicity, PAH-metabolism, and plasmid content. (D) Functional clustering of the 27 mycobacterial genomes based on COG functions. Green circles and red triangles indicate free-living and host-dependent lifestyles, respectively.

pigmentation, pathogenicity, and specific biochemical properties [57]. For the 27 mycobacterial strains, lifestyle (free-living, facultatively host-associated, or obligately intracellular), growth rate (fast- or slow-growing), and pathogenicity (non-pathogenic or pathogenic), together with PAH-degrading ability (PAH-degrading or PAH-nondegrading), all of which are fundamentally heterogeneous general phenotypes with low resolution, were used in dichotomous keys for two-group groupings and comparisons.

Traditionally, a major descriptive division of mycobacteria is related to growth rate: the fast-growers (colonies seen within 7 days) and the slow-growers (colonies not seen within 7 days) [2,3]. Out of the 27 mycobacteria, M. smegmatis [25], M. gilvum [13], M. vanbaalenii [26,27], M. abscessus [36] and Mycobacterium sp. strains MCS [12,29], KMS [12], and JLS [12] are fast-growers, whereas other mycobacteria, including $M$. avium [34,35], $M$. ulcerans [32,33], M. marinum [31], M. canettii [40], the M. tuberculosis complex (with M. bovis and M. africanum) [8,16,24,25,29,40-53], and M. leprae [37,54-56], are slowgrowers. On the basis of pathogenicity, all slow-growing mycobacteria are known to be associated with human or animal diseases, whereas the group of fast-growing mycobacteria has only one important human pathogen, $M$. abscessus [36]. Non-pathogens and fast-growers are freeliving (FL), whereas all pathogenic mycobacteria are either host-dependent, facultatively-host-associated (FHA), or obligate intracellular (OI) parasites. All mycobacteria that degrade PAHs have overlapping phenotypic features; they are fast-growing, non-pathogenic and free-living. One or more plasmids are found in many free-living mycobacteria, particularly in the PAH-degraders, and in a few 
pathogenic FHA mycobacteria, but not in the M. tuberculosis complex [16,24,25,29,40-53] or M. leprae [37,54-56].

\section{Phylogeny of the 27 mycobacterial strains}

Compared with a 16S rRNA-based tree (data not shown), despite its significant structural similarity, the evolutionary reconstruction based on the concatenation of the sequences of 22 conserved genes (in the core genome of the 27 mycobacterial genomes) substantially enhanced the vertical phylogenetic resolution (Figure $2 \mathrm{~A}$, available on TreeBASE S16971). The enhanced discriminatory power was able to segregate the pathogenic and PAH-nondegrading $M$. abscessus from the cluster of PAHdegrading strains in the tree. Therefore, the concatenated sequence-based phylogeny was in congruence with the phenotypic traits, lumping groups of mycobacterial strains together that have common phenotypic characteristics, in terms of lifestyle, growth rate, pathogenic characteristics, and PAH-metabolic capabilities (Figure 2C). The depth of phylogeny could function as a threshold to classify the genus Mycobacterium at the degree of phenotypic dichotomy. In the case of growth rate, the fast-growing mycobacteria are more deeply branched to the left of the tree, separated from the slow-growing strains, which form monophyletic clusters with a high intra-cluster relationship (Figure 2A). Here, the fast-growing but pathogenic $M$. abscessus is positioned between the fast-growing PAHdegraders and the slow-growing pathogenic Mycobacterium sp. JDM601 (Figure 2A and C). The six well-known $\mathrm{PAH}$-degrading mycobacteria are grouped into an isolated cluster embedding $M$. vanbaalenii PYR-1 (Figure 2A and $\mathrm{C}$ ). As illustrated in Figure 2A, the distinction between PAH-degraders and PAH-non-degraders can also be easily made on the threshold of the red dashed line. The 27 mycobacteria were clustered into 3 groups (M. smegmatis, the six PAH-degraders, and the twenty PAH-non-degrading pathogens), in which members belonging to the same clusters shared the same PAHdegrading metabolic capability.

Overall, the concatenation of the conserved genes enhanced discriminatory resolution of the mycobacterial phylogeny, and despite their polyphyletic and heterogeneous properties, the phenotypes as dichotomous keys for two-group groupings and comparisons function well as a reliable indicator of phylogenetic relationships in the tree, greatly facilitating the phenotypic delineation of clusters. Especially, the combination of the phylogeny and the phenotypic dichotomy provided a global view for the evolutionary process and direction of the genus Mycobacterium. Therefore, the integrated use of phenotypic and genotypic (or genomic) traits was essential for better understanding and dissecting of the heterogeneity of the phenotypes at the genomic (or pan-genomic) perspective, which is a key for tracing the evolutionary events.

\section{Pan-genomic dynamics according to the phylogeny of the 27 mycobacterial strains}

The structure and phenotypic behavior of the phylogeny for the genus Mycobacterium agree with the proposed model of bacterial evolution: from free-living organisms with larger genomes to host-dependent pathogenic organisms with smaller genomes [58,59]. Based on the observed genome alteration, it could be inferred that the genome dynamics reflected selective environmental forces, which defined the degree of diversity and dynamics of genomes corresponding to phenotypic evolution.

On the basis of the pan-genomic concept [60,61], genome dynamics according to the phylogeny of the 27 mycobacteria were measured to assess the evolutionary scale against which the evolutionary steps led to the contemporary genomes. As shown in Figure 2B and $\mathrm{C}$, the mycobacterial pan-genome shows initially 'open' but later 'closed' pan-genomic properties. The size of the pan-genome increased rapidly at the beginning from $M$. smegmatis to $M$. marinum via the six PAH-degraders ('open' pan-genome added approximately 11,686 new genes), but the number of genes added to the pan-genome was comparatively much smaller with the genomes of pathogenic strains of the $M$. africanum, $M$. bovis, and $M$. tuberculosis clade ('closed' pan-genome added only 1,363 genes). On the other hand, the core-genome dropped significantly in size after the addition of the first PAH-degrader Mycobacterium sp. MCS (loss of 2,973 genes) and the first pathogen, $M$. abscessus (loss of 1,005 genes). Another noticeable drop was observed in the OI parasite $M$. leprae genome (loss of 425 genes), which is consistent with the phenotypic changes. Interestingly, M. abscessus, M. avium, Мycobacterium sp. JDM601, M. ulcerans, and M. marinum were scattered away from the stable clusters of the six $\mathrm{PAH}$-degrading mycobacteria and the $12 \mathrm{M}$. tuberculosis complex strains (Figure 2A and D). The mycobacteria ( $M$. abscessus, M. avium, Mycobacterium sp. JDM601, $M$. ulcerans, and M. marinum) showed a continuous, rapid pan-genome growth, which was followed by a relatively stable size of core-genome after the transient decline by $M$. abscessus. On the contrary, the $12 \mathrm{M}$. tuberculosis complex strains, with close phylogenetic relationships, showed stable sizes of pan- (only avg. 110 genes increasing) and core-genomes (only avg. 10 genes decreasing), leading to a 'closed' pan-genome. The core-genome and pan-genome for all 27 mycobacterial genomes were estimated to be approximately 925 and 20,342 genes, respectively.

In pan-genomic increase, the six PAH-degraders showed an 'open' pan-genomic property, a continuous and gradual pan-genome growth, which added approximately 5,320 new genes. The addition of the first Mycobacterium sp. 
MCS to the genome of the PAH-non-degrading M. smegmatis contributed 1,870 new genes to the pan-genome. With the five PAH-degrading genomes, the pan-genome grew by an average of $690( \pm 367)$ genes every time a new genome was added. On the other hand, after the first Mycobacterium sp. MCS caused a significant drop (loss of about 2,973 genes) in the core genome, additional genomes of the PAH-degrading mycobacteria had a minimal effect on the core-genome, which indicates that the coregenome size is relatively static. Since the genomes are similar in size, a continuous and rapid pan-genomic increase while maintaining a stable core-genome indicates a high variability of dispensable (found in one or more but not all genomes) parts of the genomes of PAH-degrading mycobacteria. Overall, the pan-genomic features of the PAH-degraders, the apparent drop of the core-genome in size and the continuous and rapid growth of the pangenome agree well with their phylogenetic and phenotypic isolation in the genus Mycobacterium.

\section{Functional clustering analysis based on the genomic contents of the $\mathbf{2 7}$ mycobacterial strains}

To visualize the function-based genomic comparison of the 27 mycobacteria, which evolve through vertical inheritance and horizontal gene transfer (HGT), genomes were clustered based on similar functional profiles. In general, as shown in the principal components analysis (PCA) clustering (Figure 2D), the vertical phylogenetic relevance was well conserved in the cluster structure, based on the genomic clusters of orthologous groups (COG) content distance, and enriched the genome-scale perspective of lateral evolution at a functional level. In addition, the proximity of clustering accounted well for not only the pan-genomic dynamics but also the phenotypic features of the 27 mycobacteria at the genus level. For instance, when clustered by the blue threshold line in the phylogenetic tree (Figure 2A), the clade of $20 \mathrm{PAH}$-non-degrading pathogenic mycobacteria was further divided into 7 clusters, which provides enhanced discriminatory resolution, reflecting a transitional stage in terms of phenotype and pan-genome evolution. In the functional cluster structure, despite the conserved phenotypic spectrum, six mycobacteria, M. abscessus, M. avium, M. ulcerans, M. avium subsp. paratuberculosis K-10, Mycobacterium sp. JDM601, and $M$. marinum, were dispersed clearly away from the stable clusters of the six PAH-degraders and the M. tuberculosis complex and M. leprae, the OI parasites (Figure 2D).

Consistent with the phylogenetic affinity and pangenomic properties, the six PAH-degrading mycobacteria were clustered together, indicating that they share similar functional genomics inventories. Interestingly, despite its significant vertical phylogenetic distance and pan-genomic difference, the PAH-non-degrading M. smegmatis was also in close proximity to the cluster of four PAH-degrading mycobacteria (strains KMS, PR-GCK, PYR-1 and MCS). The closer proximity of the PAH-non-degrader to four of the PAH-degraders than to the other PAH-degrader, Mycobacterium sp. JLS, indicates a high degree of functional genomics similarity between $M$. smegmatis and the four PAH-degrading strains (Figure 2D) and, as revealed by the following genomic analysis, $M$. smegmatis also had several genes involved in PAH degradation. The observed functional genomics distance suggests that the PAH-degraders share similar genetic and physiological backgrounds for successful microbial metabolism of PAHs, which is apparently an attractive phenotype to mycobacteria in an oligotrophic habitat highly contaminated with PAHs [21].

\section{Reconstruction of a mycobacterial phenotype network (MPN) for the genus Mycobacterium}

Phenotype networks (PNs) describe the interconnectivity among phenotypic traits. They have been shown to be important in better understanding complex epistatic and pleiotropic behaviors of biological systems from a global perspective [23]. To decipher the genomic epistasis and pleiotropy for mycobacterial phenotypic evolution in terms of PAH degradation, we first reconstructed an MPN and then performed sequential functional pan-genomic comparison, using the phenotype nodes to elucidate emerging properties of the MPN as a whole. The MPN was reconstructed from vertical phenotypic interrelationships as well as horizontal phenotype dichotomy, which accounts for the mutual effects on phenotypic evolution (Figure 3).

The topological structure of the MPN clearly indicates that the mycobacterial phenotypes are linked with scalefree features: (i) preferential linking among phenotype nodes and (ii) a strongly connected hub phenotype node. In the MPN, all phenotype nodes apparently show lifestylecentric preferential connections, reflecting their phenotypic relationship; three nodes, 'PAH-degrading, 'Fast-growing', and 'Non-pathogenic' are connected to each other with the center at the 'Free-living (FL)' node, whereas the other three nodes, 'Pathogenic,' 'PAH-non-degrading', and 'Slowgrowing' are linked to each other with centers at the 'facultatively-host-associated (FHA)' and 'obligately intracellular (OI)' nodes (Figure 3). In the MPN, most phenotypes showed strong connectivity, with the exception of the phenotype 'Fast-Growing'. The phenotype 'Fast-growing' is strongly linked to the 'Free-living (FL)'-centric nodes ('PAH-degrading' and 'Non-pathogenic') but has a weak connection to the phenotypes, 'FHA' and 'Pathogenic'. The weak connection of the phenotype node 'Fast-growing' toward 'FHA' and 'Pathogenic' caused by M. abscessus, which is a fast-growing but facultatively-host-associated pathogenic mycobacterium. Together with M. abscessus, medically important fast-growing pathogenic mycobacteria 


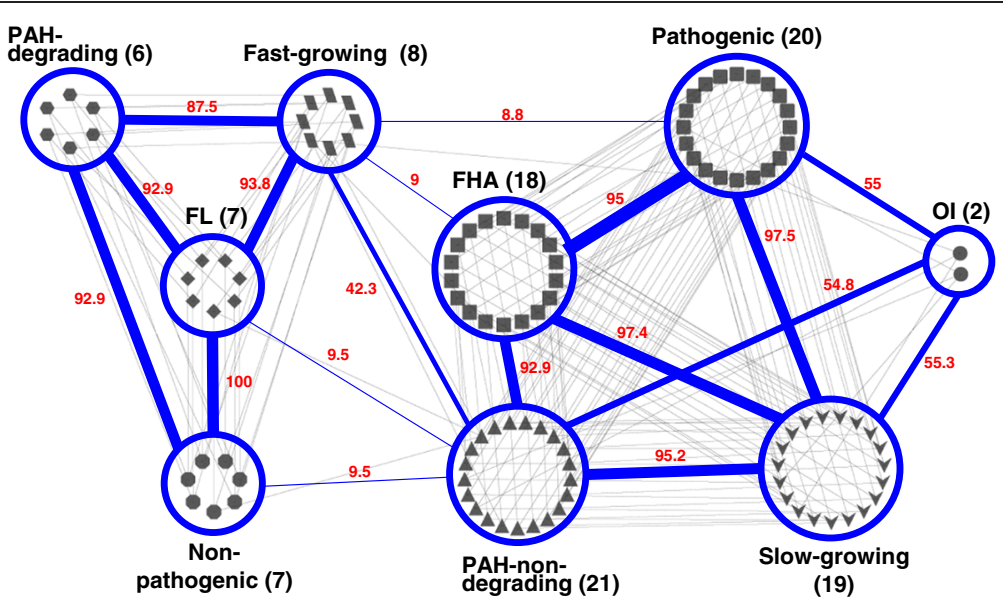

Figure 3 Mycobacterial phenotype network (MPN) of the $\mathbf{2 7}$ mycobacteria. The number of mycobacteria belonging to the phenotype is given in brackets after each name of a phenotype node (blue ellipse). In the MPN, the node shapes of the 27 mycobacterial strains were represented based on the 9-phenotype classification as explained in the text: diamond, FL; hexagon, PAH-degrading; octagon, Non-pathogenic; parallelogram, Fast-growing; rectangle, FHA; triangle, PAH-non-degrading; round rectangle, Pathogenic; v, Slow-growing; ellipse, OI. Together with the numerical connection strength score (in red), the thickness of the connection lines (edge, blue) among the phenotypes indicates the degree of the connection strength. In the MPN, the phenotypes FL and Non-pathogenic show the highest connection strength [CS (Gx, Gy), 100], while Fast-growing (8) —Pathogenic (20), Fast-growing (8) — FHA (18), FL (7) —PAH-non-degrading (21), and Non-pathogenic (7)—PAH-non-degrading (21) show weak connection strength $[8.8<\mathrm{CS}(\mathrm{Gx}, \mathrm{Gy})<9.5]$.

are essentially limited to $M$. chelonae and M. fortuitum, which are associated with traumatic and surgical wound infections, skin and soft tissue infections, and pulmonary disease [62]. It should be noted that the weak connection of the node 'PAH-non-degrading' with the 'Fast-growing' and 'Non-pathogenic' nodes resulted from narrow observation caused by the limited number of available mycobacterial genomes. As revealed in the comprehensive topological structure of the MPN, therefore, the phenotype 'Fastgrowing' shows a transitional phenotypic relationship, bridging the different lifestyle-centric phenotype nodes, which is consistent with its phylogenetic and functional positions.

Another interesting finding was that the phenotype 'PAH-non-degrading' is a hub phenotype in the MPN with the highest connection degree of 7 (Figure 3). Consistent with the highest phenotypic compatibility of the hub node, its dichotomy phenotype 'PAH-degrading' is a relatively specific and isolated phenotype in the MPN. The 'PAH-degrading' node has the lowest connection degree of 3 and an apparently biased connection toward the other two FL-centric phenotypic nodes, 'Fast-growing' and 'Non-pathogenic'. That is, the 'PAH-degrading' node has no connection with the phenotypes 'Pathogenic' and 'Slowgrowing, which are the main phenotypic traits of the 'FHA' and 'OI' mycobacteria. These connection features of the 'PAH-degrading' node in the MPN suggest that PAH degradation might be an isolated specific phenotype, limited to a phylogenetically isolated group of mycobacteria.

Overall, together with a useful mycobacterial compendium of phenotypic and genomic properties (Figure 2), the apparent lifestyle-dependent phenotypic connection of the MPN provides global insights into the direction of phenotypic evolution, including PAH degradation, in the genus Mycobacterium. In addition, network features of the 'PAH-degrading' phenotype in the MPN suggest a relatively low degree of robustness in the face of genetic and environmental changes.

\section{Pan-genomic perspective of the MPN}

The pan-genomic comparison (core- and pan-genomes) of the phenotypic nodes not only further supports the interrelationships and stability of the phenotype nodes in the MPN but also allows better understanding of the relative roles of the core- and dispensable-genomes in phenotype evolution. For quantitative comparison of the phenotypic nodes, we extended the pan-genomic concept to the MPN. As revealed in the pan-genomic comparison (Figure 4A), the 'Free-living (FL)'-centric phenotype nodes have a relatively large core-genome but a small dispensable-genome compared to the 'FHA'- and 'OI'centric nodes. For instance, the seven sympatric mycobacteria belonging to the 'FL' node have a relatively large genome size and core-genome than those of the two allopatric strains in the 'OI' node. In addition, there is an association between connectivity of the phenotype nodes and their pan-genomic size, in which the phenotypic nodes with strong connectivity in the MPN show a similar pan-genome size (Figure 4A). The three nodes 'PAHdegrading,' 'Free-living (FL)' and 'Non-pathogenic' show a similar pan-genome to core-genome ratio, which are 9,532/3,533 for the 'PAH-degrading' node and 


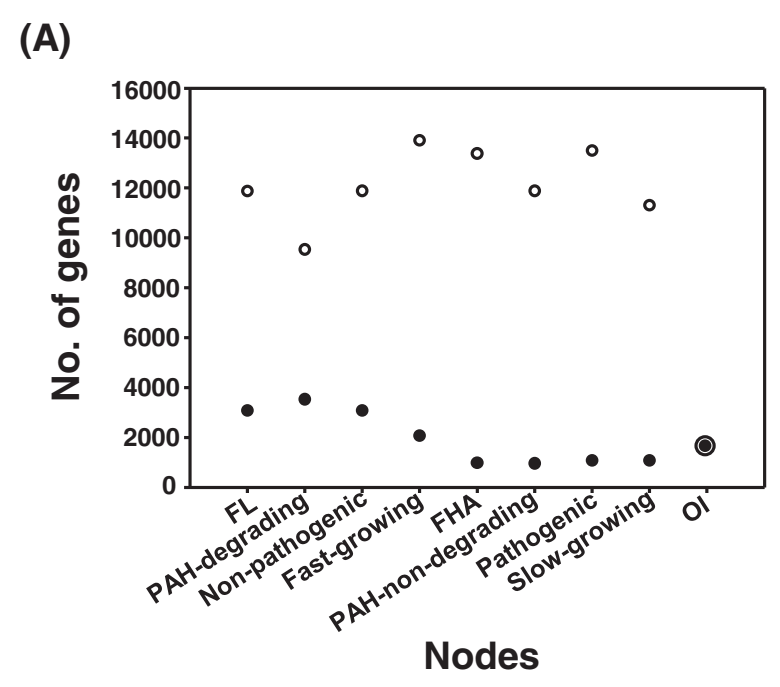

(B)

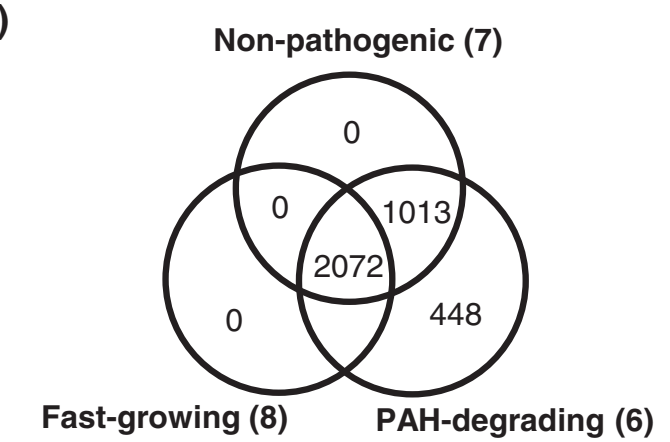

Figure 4 Comparative pan-genomics based on the MPN. (A)

Pan-genomic comparison (core- and pan-genome) of the phenotype nodes in the MPN. The open and closed circles indicate the number of genes of the pan-genome and core-genome of each phenotypic node, respectively. The pan- (open circle) and core-genome (closed circle) of the phenotype node Ol overlapped. (B) Venn diagram analysis of the core genomes of the three phenotype nodes in the FL-centric sub-MPN.

$11,878 / 3,085$ for the 'FL' and 'Non-pathogenic' nodes. The 'Fast-growing' is an exception to this, having a relatively small core-genome of 2,072 and a large pan-genome of 13,909 , which is similar to those of the FHA-centric nodes. The FHA - and OI-centric nodes, 'PAH-nondegrading, 'Pathogenic', and 'Slow-growing', have significantly bigger pan-genomes but smaller core-genome ratios than those of the 'FL'-centric nodes, which are 13,541/958 genes for the 'PAH-non-degrading', 13,541/983 genes for 'Pathogenic', and 11,308/1,077 genes for 'Slow-growing'. As a result, all FHA - and OI-centric phenotypic nodes have relatively small core-genomes but large dispensable genomes, which indicate a high degree of genome diversity of the phenotype node. Most of all, the bigger coregenome size of the 'FL'-centric nodes than those of the 'FHA'- and 'OI'-centric nodes results from both higher numbers of genes per genome and genomic similarity. It suggests that the relatively small core-genome and large pan-genome of the 'Fast-growing' node definitely resulted from inclusion of $M$. abscessus, which is distant from other free-living mycobacteria in terms of phylogeny and phenotype.

In the 'PAH-degrading' node, the pan-genome contains 9,532 genes, of which 3,533 genes belong to the coregenome that is present in each strain and 5,999 genes belong to the dispensable genome that is absent in one or more strains (Figure 4A). Except for the node OI, the phenotype 'PAH-degrading' node shows the smallest pan-genome and dispensable genome and the largest core-genome. This pan-genomic feature clearly suggests a high degree of genomic similarity between members of the PAH-degraders and the core-genome with unique genes, essential for the 'PAH-degrading' phenotype. A Venn diagram analysis using the core-genomes of the 'FL'centric phenotype nodes revealed that the 'PAH-degrading' node has several hundred unique genes (Figure 4B). Out of 448 unique genes, about 34 genes encode the catabolic enzymes involved in RCP (18, 53\%), SCP (12, 35\%), and CAP $(4,12 \%)$ that are responsible for PAH metabolism in $M$. vanbaalenii strain PYR-1, whereas about 415 genes encode enzymes for diverse cellular functions, such as 143 genes (35\%) for cellular metabolism (MET), 44 genes (11\%) for information storage and processing (ISP), 57 genes (14\%) for cellular processes and signaling (CPS), and 90 genes (22\%) that are poorly characterized. Consistent with the pan-genomic properties of the 'PAH-degrading' node, its dichotomy phenotype node, 'PAH-non-degrading", shows the smallest core-genome of 958 genes and largest pangenome of 13,541 genes. The pan-genomic features of the 'PAH-degrading' node are consistent with the monophyletic affinity in phylogeny and the biased connection preference to the phenotype nodes ('FL', 'Non-pathogenic', and 'Fast-growing' nodes) with similar pan-genomic sizes in the MPN.

\section{Functional pan-genomic view of the six PAH-degraders with PAH-degrading phenotype in the MPN}

For functional pan-genomic understanding of the PAHdegrading phenotype, we investigated the pan-genome of the six PAH-degrading mycobacteria in the 'PAH-degrading' node based on the previous proteomic data from M. vanbaalenii PYR-1 [9]. As shown in Figure 5A and Additional file 1: Table S1-S5, we categorized the pan-genome of 9,532 genes from the six strains, among which 3,533 genes were considered to be core genome and 5,999 genes were dispensable genome. The core-genome accounted for $37 \%$ of the pan-genome and, as shown in functional analysis, shows a distribution across all COG categories (Figure 5B; Additional file 1: Tables S1 and S2).

Among the 3,533 core genes, 136 genes were tentatively identified to be involved in the degradation of aromatic hydrocarbons (Additional file 1: Table S3). According to 


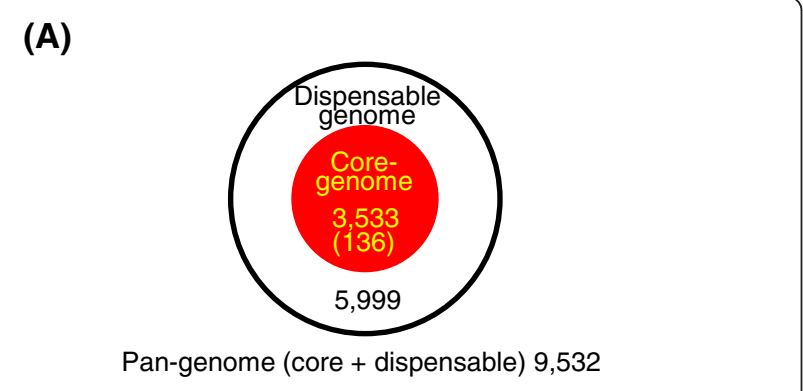

(B)

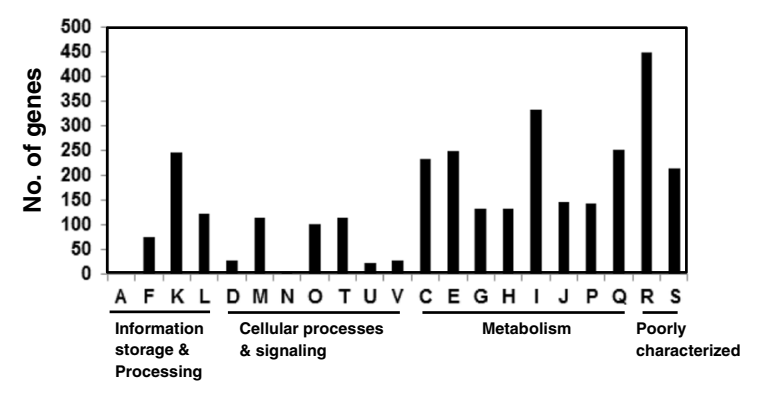

(C)

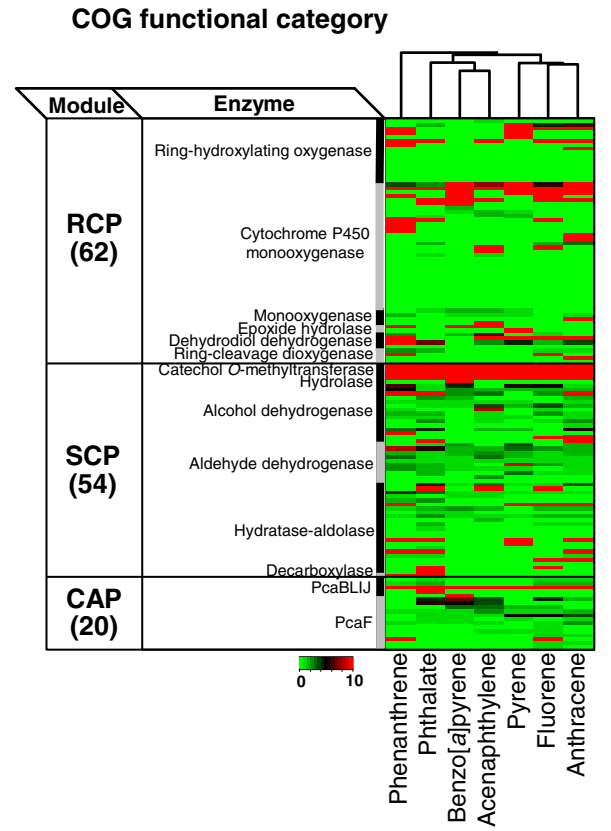

Figure 5 Systematic genome analyses of the six PAH-degrading mycobacteria. (A) Pan-genome of the six PAH-degrading mycobacteria. (B) Functional classification of the 3,533 core-genome based on COG category. (C) Classification of the 136 catabolic enzymes based on functional module and enzyme function in PAH-MN. Proteomic expression patterns under different aromatic hydrocarbon treatments are also shown.

the functional module proposed in the PAH-MN in $M$. vanbaalenii PYR-1 [9], about 62 genes, involved in the functional module RCP, made up the largest portion of the list of 136 genes, which is followed by 54 and 20 genes, involved in SCP and CAP, respectively (Figure 5C;
Additional file 1: Table S4). Especially, as shown in the proteome data (Figure 5C), about 113 genes out of the 136 genes are expressed as proteins involved in $\mathrm{PAH}$ degradation pathways (Additional file 1: Table S5) and the expression of these proteins is apparently $\mathrm{PAH}-$ substrate-regulated. Therefore, this functional genomics observation not only clearly indicates that the proteins of the core genome function for PAH-metabolism but also provides some insights into the genetic requirements and metabolic potential in terms of PAH degradation; they share similar genetic repertoires and $\mathrm{PAH}$ metabolic spectra.

\section{Contribution of HGT events to the PAH-degrading phenotype}

Most free-living mycobacteria are naturally competent, that is, they can take up genetic material from the environment and recombine it into their chromosome [63]. Analysis of the genome sequences of the PAH-degrading mycobacteria revealed many horizontally acquired DNA regions (genomic islands [GIs]) interspersed in their genomes. These GIs are mostly larger than $4 \mathrm{~kb}$ in length (4 $20 \mathrm{~kb}$ in size), with average numbers being $35.8( \pm 7.5)$ per genome (data not shown). The number of HGT genes identified in the PAH-degrading mycobacteria ranged from 437, in M. vanbaalenii PYR-1, to 536, in M. gilvum PYR-GCK (average of $480 \pm 40$ ), and is 2,878 in total (Figure 6A; Additional file 2: Table S6). Among these HGT genes, we identified 754 and 2,124 genes that contribute to the core-genome (a total of 3,533 genes) and the dispensable genome (a total of 5,999 genes), respectively (Figure 6B; Additional file 2: Tables S7-8). Interestingly, the contribution of the horizontally transferred 754 genes to the 5 COG cellular metabolism categories, $\mathrm{C}, \mathrm{E}$, F, G, H, and I, was 339 genes (53\%) (Figure 6C), which is apparently higher than that seen in the genomes of PAHdegrading mycobacteria (around 30\%). It indicates that GIs contribute to the metabolic proficiency of these organisms, including the ability to degrade PAHs.

Considering only $\mathrm{PAH}$ degradation, we identified as horizontally transferred 29 genes, which are enough to cover all the enzymatic reactions in the general scheme of PAH degradation proposed for M. vanbaalenii PYR-1 (Figure 6D; Additional file 2: Tables S9-10) [9]. Many PAH-catabolic genes identified on GIs were also located on the plasmids of PAH-degrading mycobacteria, such as Mycobacterium spp. MCS and KMS, which were isolated from geographically different locations. Comparison of GIs and PAH-catabolic genes among these PAH degraders showed conservation of $\sim 150-\mathrm{kb}$ catabolic gene clusters, with slightly different genetic configurations (data not shown). Together with the pan-genomic evidence, it strongly demonstrates that most PAH-degrading strains share the same genetic sources and pathway evolution by patchwork assembly (assembly of existing pathways in 
new combinations), which has been systematically evaluated as highly successful [9]. It appears that the HGT events of the highly conserved PAH-degrading gene clusters could provide practical benefits: (i) overcoming evolutionary selective pressure, (ii) acceleration of the buildup speed, and (iii) metabolic maximization from the limited genetic modules $[9,64]$.

Unique genes in PAH-degrading core-genome but not the pan-genome of $\mathrm{PAH}$-non-degraders

Under dynamic HGT events, the PAH-degrading phenotype might be equally open to all mycobacteria. As expected from the pan-genomic view, however, the 'PAH-degrading' HGT events do not have the same metabolic effects on all competent mycobacteria, because of several types of influences, including environment and stochastic, molecular or epigenetic variation among individuals $[58,65,66]$. However, other major influences on HGT events could be the genetic backgrounds of competent mycobacteria. To understand the unique genetic backgrounds related to the PAH-degrading phenotype, a comparative analysis of the core-genome of the 'PAH-degrading' node and the pan-genome of the 'PAHnon-degrading' node was conducted. It led us to find

(A)

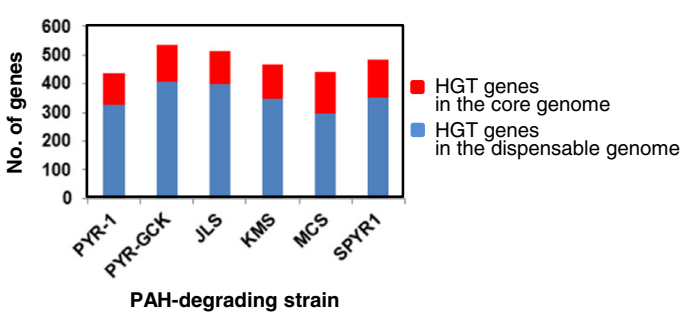

(B)

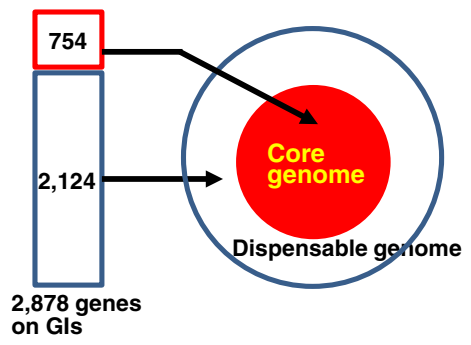

(C)

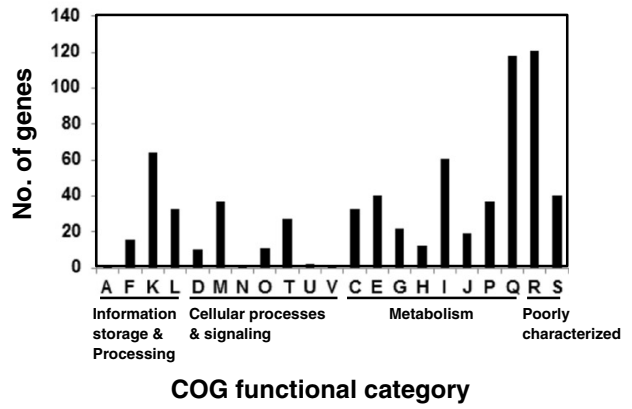

(D)

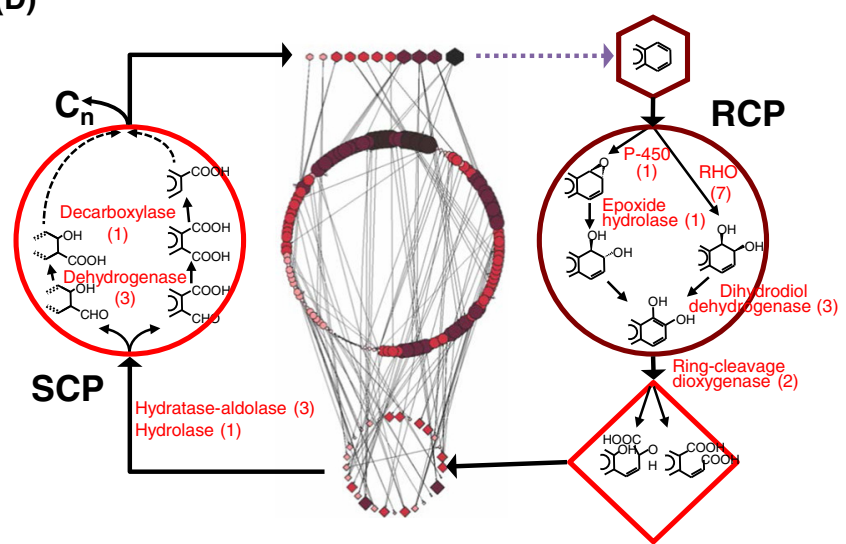

Figure 6 Genomic islands (Gls, large-genomic regions that contain multiple genes of probable horizontal origin) analysis of the PAH-degrading mycobacteria. (A) Comparison of the Gls from the PAH-degrading mycobacteria, depending upon core and dispensable genes. (B) HGT contribution of the 2,878 Gl-located genes to the pan-genome of PAH-degrading mycobacteria. (C) Functional distribution of the 754 horizontally transferred genes to the core-genome based on COG. (D) Functional annotation of the horizontally transferred PAH-catabolic genes with respect to the general scheme of PAH metabolism proposed for M. vanbaalenii PYR-1. Only the 22 genes involved in RCP and SCP are shown. Please see reference [9] for a more detailed explanation of the general scheme of mycobacterial PAH metabolism. 
unique genes, which might be directly or indirectly related to PAH metabolism, in all PAH-degrading mycobacteria. As shown in Figure 7A and Additional file 3: Tables S11-12, about 248 unique genes were identified only in the core-genome of the PAH-degrading mycobacterial genomes. From the list of 248 unique genes, 154 genes (62\%) were categorized based on COG function or functional modules in the PAH-MN, among which 35 genes were assigned to RCP (23 genes), SCP (10 genes), and CAP (2 genes) for PAH metabolism [9]. The other 119 genes were assigned to other cellular functions (Figure 7B; Additional file 3: Tables S11-12). Interestingly, 46 genes (19\%) of the unique gene pool were identified as horizontally transferred. When analyzed in terms of protein expression, 146 (59\%) genes were expressed under all experimental conditions, with 28 (61\% of 46 genes) horizontally transferred genes.

Out of the 23 proteins assigned to the RCP functional module in the PAH-MN, 12 genes encode oxygenase components for six ring-hydroxylating oxygenase (RHO) enzyme systems $[9,67]$. Six of the 12 genes encode the RHO components, NidAB, NidA3B3, and PdoA2B2, which belong to a type $\mathrm{V}$ RHO system, while the other 6 genes encode RHO enzymes belonging to a type X RHO system, based on Kweon's RHO classification [67-69]. Notably, these 3 RHOs, NidAB, NidA3B3, and PdoA2B2, have been shown to be the main enzymes involved in the initial oxidation of HMW PAHs, such as pyrene and fluoranthene, and LMW PAHs, such as phenanthrene, anthracene, and fluorene [5-8,14,15,18,19,67,68,70,71].

Overall, it appears that the conserved unique gene pool contained only in the PAH-degraders was obtained mainly by HGT events and plays crucial roles in the $\mathrm{PAH}$-degrading phenotype, either directly by the PAHdegrading enzymes, such as the three type V RHO systems in the RCP functional module, or indirectly by the 120 proteins with versatile cellular functions. As revealed in the following gene evolutionary analysis using the Count software [72], considering the significant contribution of HGT events to the conserved unique genes, the uniqueness of the PAH-degradation genes identified only in the PAH-degraders might be explained by gene loss during evolution of the PAH-degrading phenotype under selection. When compared with the core-genome of the PAH-degraders, the unique gene pool also indicates that PAH-non-degraders also have a significant number of genes directly involved in PAH metabolism, which might reveal how closely the PAH-degraders are related to PAHnon-degraders.

The presence, organization, and dynamics of PAH-degrading genes in the genus Mycobacterium

To explain the PAH-degrading phenotype and its evolution in the genus Mycobacterium, the presence (Figures 8
(A)

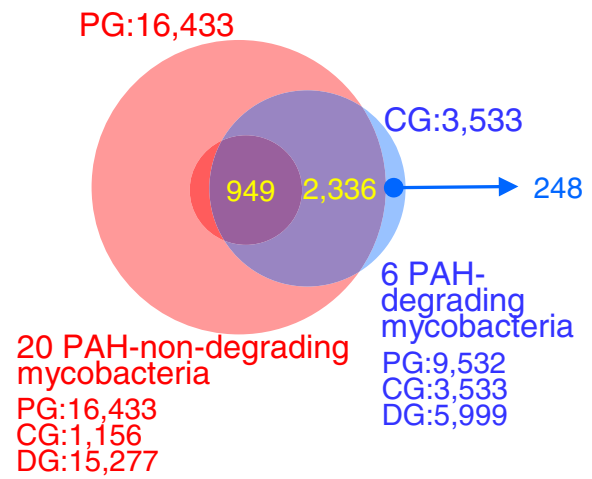

(B)

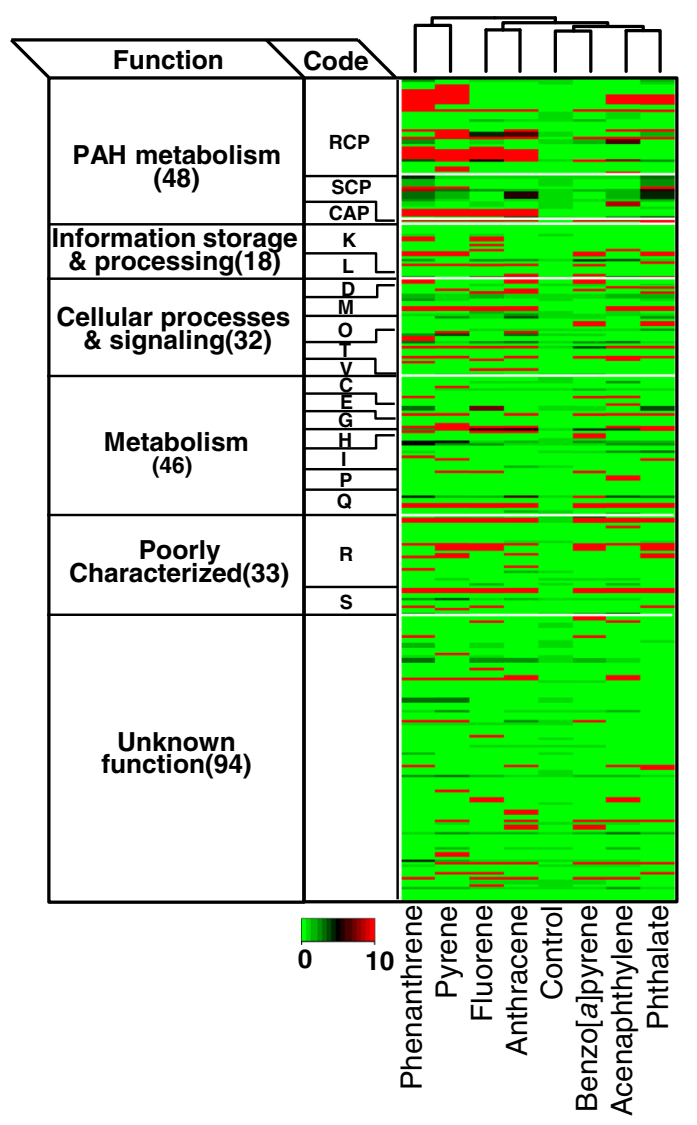

Figure 7 Identification of the genes found only in the six PAHdegrading mycobacteria. (A) Comparison of the core genome of the six PAH-degrading mycobacteria and pan-genome of the 20 PAH-non-degrading mycobacteria. (B) Functional classification and their regulation of the unique genes in the core genome based on the COG functional category. Please see reference [9] for the detailed protein expression information.

and 9), organization (Figure 10), and dynamics (gain/loss, Figure 11) of the PAH-degrading genes, essential for the complete metabolism of PAHs, were analyzed. To address the distribution of PAH-degrading genes and capabilities throughout the genome of mycobacteria, the PAH- 


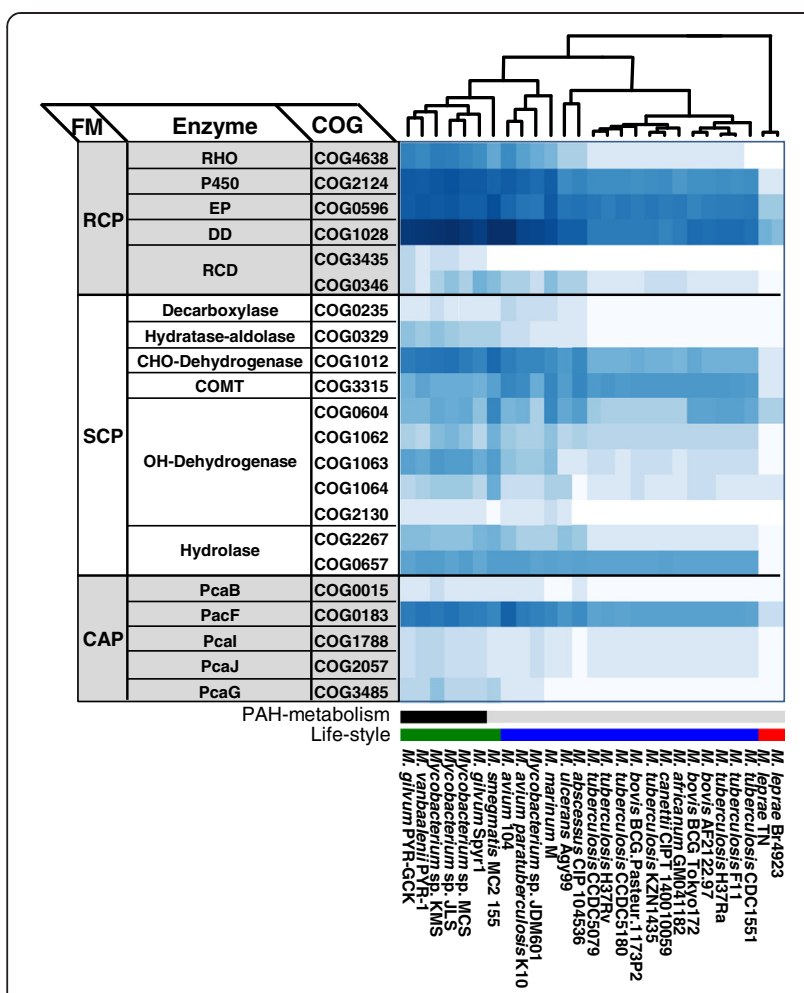

Figure 8 Distribution of PAH-degrading genes across the genus Mycobacterium. The genes involved in the PAH-MN were classified based on functional modules and COG functional categories. In the cluster analysis of the heat map, the presence/absence pattern of the PHA-degrading genes shows apparent relationships with the PAH-degrading abilities and lifestyles of the 27 mycobacteria. The six PAH-degraders were grouped together in the left, forming a cluster separated from PAH-non-degraders.

degrading genes based on functional modules of the PAH-MN were surveyed [9]. Interestingly, as revealed in the analysis for the unique genes (Figure 7), most putative genes for $\mathrm{PAH}$-degradation are widespread in the genus Mycobacterium. The PAH-degrading genes identified were between 37 (M. leprae Br4923) and 425 genes (Mycobacterium sp. JLS) from the genomes of the 27 mycobacteria (Additional file 4: Table S13). Although overall distribution of the catabolic genes among the mycobacteria is variable, the clustering results clearly show a correlation between the gene profiles and the PAH-degrading ability. As shown in the overall view of the heat map (Figure 8), the six PAH-degraders were clustered together to the left, separated from the "PAHnon-degrading" strains, suggesting genomic differences in terms of PAH-degradation gene distribution. In addition, there is an apparent gradual decrease in the number of genes during evolution from 'FL' to 'OI' via 'FHA' mycobacteria, with several genes showing apparent gain/loss patterns according to PAH-degrading ability (Figures 8 , 9, 10, and 11).
In the PAH-MN, the functional module $\mathrm{RCP}$, producing catechols and ring-cleavage products from $\mathrm{PAH}$ substrates for the following SCP or CAP, is a crucial process that determines substrate range and pathways [9]. A set of enzymes, including ring-hydroxylating oxygenases (RHOs), cytochrome P450 monooxygenases (CYPs), epoxide hydrolases, cis-dihydrodiol dehydrogenases, and ringcleavage dioxygenases (RCDs), is responsible for RCP [9]. Among them, two groups of oxygenases, CYPs and RHOs, are known to initiate mycobacterial PAH degradation by ring mono- or di-hydroxylation to form transand cis-dihydrodiols, respectively $[5,8,9,14,15,70,71,73]$. In the RHOs, which are the main oxygenases for initial PAH oxidation, genome analysis of the 27 mycobacteria predicted the existence of a total of 246 genes encoding this RHO enzyme, with a range between 0 in $M$. leprae and 28 in Mycobacterium sp. KMS (Figure 9; Additional file 5: Table S14). As illustrated in Figure 9A, out of the 246 RHO enzymes based on Kweon's classification [67,69], 44 RHO enzymes were assigned to type II (6 RHOs), type IV (2 RHOs), and type V (36 RHOs), and the rest (202 enzymes) were assigned to type $\mathrm{X}$ (unclassified RHO) $[67,69]$. The 36 type V RHO systems are dominant and mostly concentrated in the PAH-degraders, which normally have at least four or more type V RHO systems (Figure 9B). In particular, 29 type V RHOs out of 32 are only present in the PAH-degraders (Figure 9A and B). As revealed by the gene gain/loss analysis (Figure 11), the fact that the three type V RHOs, two Nid (nidAB and nidA3B3) systems and one Pdo (pdoA2B2) system, are consistently present (gained) in the PAH-utilizing mycobacteria and absent (lost) in PAH-non-degraders, further supports their functional importance in PAH metabolism $[6,7,9,15,70,71]$. Interestingly, together with the 3 type V RHOs, another type V RHO, a Pht system (phtAaAb), encoding a phthalate dioxygenase, is also consistently present in the six PAH-degraders as well as in several PAH-non-degraders, such as Mycobacterium sp. JDM601, M. avium 104, and M. avium subsp. paratuberculosis K-10 (Figures 9 and 10A). A comparison of the operons containing the Pht system, however, shows that the pht operons from the PAH-non-degraders do not have two genes, phtAc and phtAd, that encode electron transfer components (ETCs), a [3Fe-4S]-type ferredoxin and a GR-type reductase, respectively (Figure 10A). The two genes phtAc and phtAd comprise a typical type $\mathrm{V}$ ETC, which is functionally compatible with type V RHO systems $[9,67,68]$. The absence of the type V ETC suggests that this incomplete Pht system could not function in the hydroxylation of phthalate, which is one of the main hub intermediates in the PAH-MN of M. vanbaalenii $[8,9]$. Furthermore, the significant numerical imbalance between oxygenase components and ETCs, the functional loss of type V ETC, which has a high degree of compatibility, 


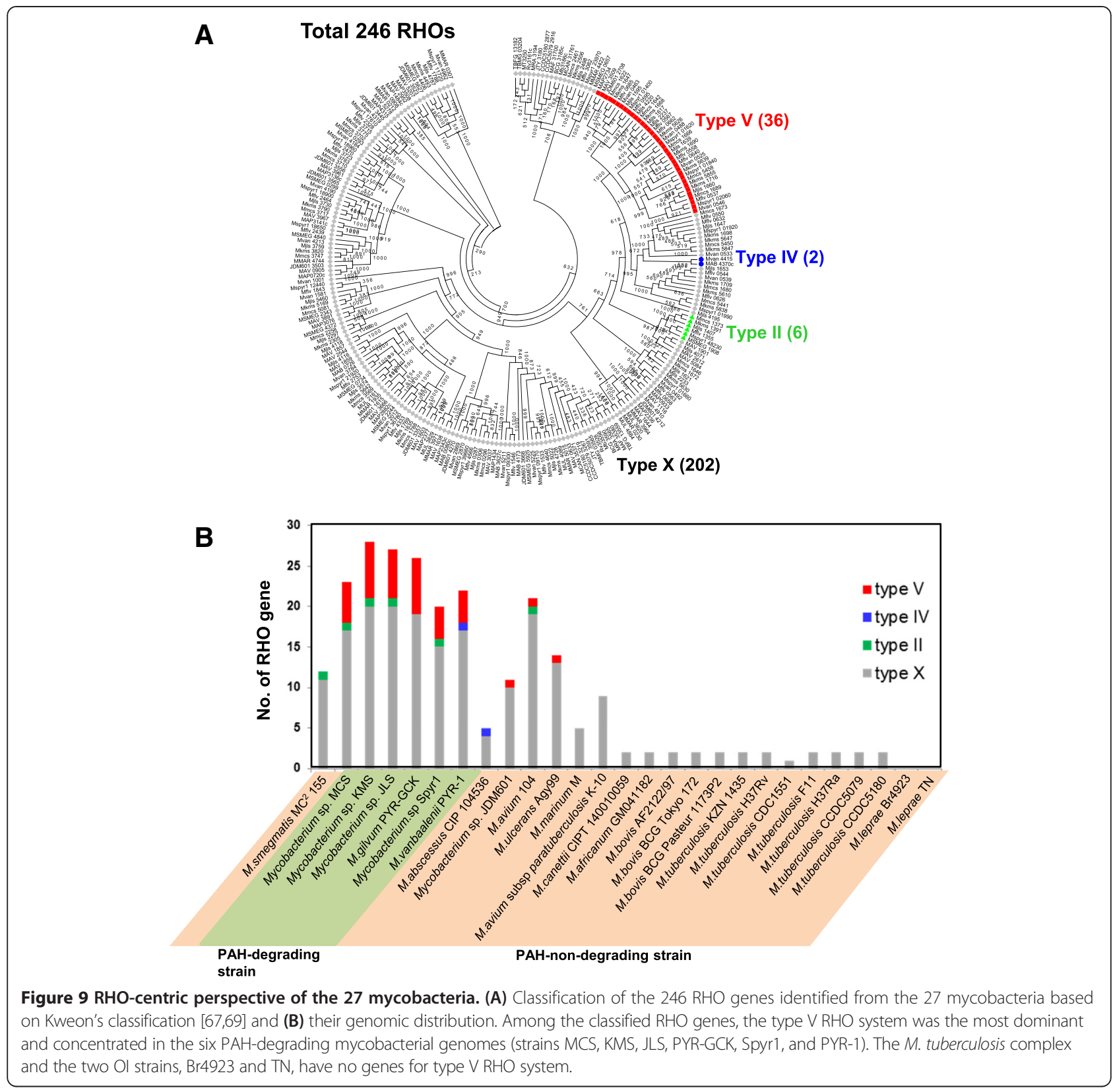

most likely hinders PAH degradation in these PAH-nondegraders. In the genome of $M$. vanbaalenii, 21 genes were identified to encode large subunit components of RHOs, whereas only one copy of the type V ETC was identified $[67,68]$. Interestingly, RHO enzymes also gradually decrease in numbers during evolution from 'FL' to 'OI' via 'FHA' mycobacteria (Figures 9 and 11). In contrast, the number of CYPs varies among 27 mycobacterial strains, which means no correlation with PAH-degrading ability of the strains (Figure 8). However, although few of their physiological roles are known, previous studies have proved the involvement of CYP enzymes in the initial oxidation of PAHs as an alternative $\mathrm{PAH}$ oxygenation reaction [73]. Among the genes involved in RCP, the gene encoding RCDs (COG3435) showed the lowest gene copy number and only the six PAH-degraders had this RCD gene (Figure 8).

SCP includes the steps that are responsible for hydrolysis of the $\alpha$-keto side chain of the ring-cleaved compounds, forming biological precursors, such as pyruvate, and, at the same time, metabolites with an aldehyde group, which should be removed during oxidation/decarboxylation in the subsequent reaction [9]. As shown in Figure 8, functionally diverse enzymes are included in this functional module. Generally these enzymes show a significant numerical abundance with minor exceptions, enabling SCP 


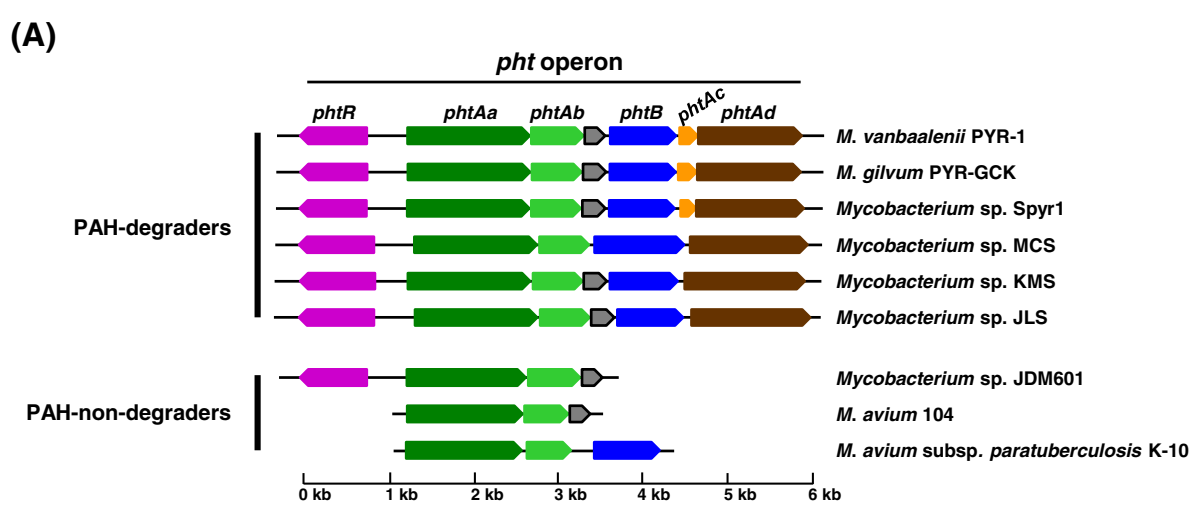

(B)

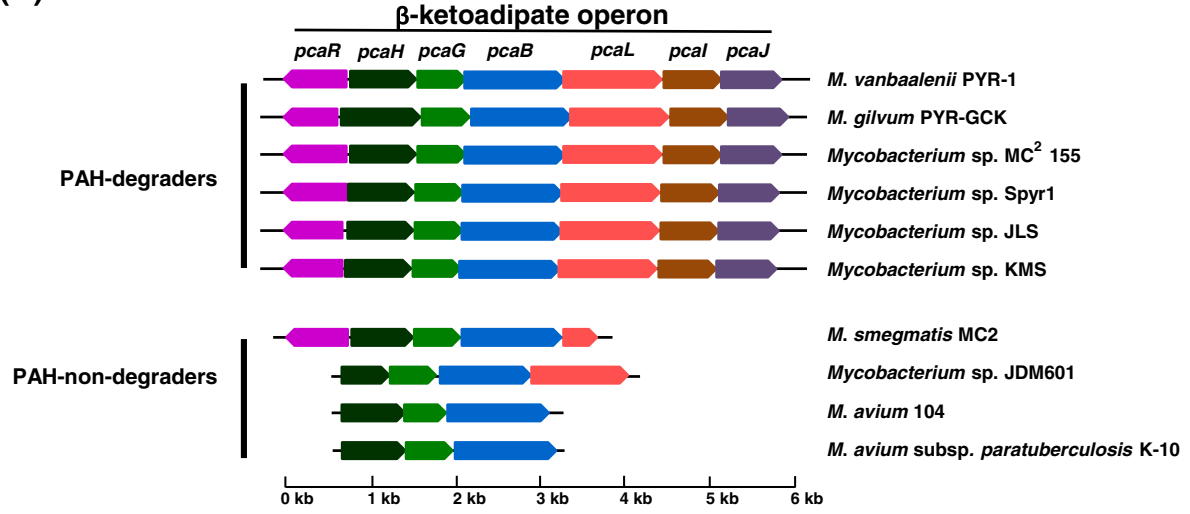

Figure 10 Comparison of the phthalate operon (A) and the $\beta$-ketoadipate operon (B) in the genus Mycobacterium including the six PAH-degraders and the PAH-non-degraders. The pht and $\beta$-ketoadipate operons, which link the peripheral pathways (RCPS and SCPS) to the TCA cycle in the PAH-MN, are conserved in the PAH-degraders, whereas some of the PAH-non-degraders, including strains JDM601, 104, and K-10, have a broken operon structure.

to accept an enormous range of ring-cleavage compounds during PAH metabolism $[8,9,18,19]$. Two enzymes, the decarboxylase (COG0235) and alcohol dehydrogenase (COG2130), are the exceptions to this observation; only one or two copies have been found in the genomes of PAH-degraders and a few PAH-non-degraders (Figure 8) [9]. Hence, despite these two enzymes' low gene redundancy, their practical functional contribution to diverse substrates with different sizes and architecture in the PAH-MN accounts for their substrate diversity and relaxed regulation $[6,7,9,68]$. Interestingly, together with an alcohol dehydrogenase (COG1062, OH-DH2), the alcohol dehydrogenase (COG2130, OH-DH4) has been lost during evolution from 'FL' to 'FHA' or 'OI' mycobacteria (Figure 11). On the contrary, genes encoding catechol-O-methyltransferase (COMT; EC 2.1.1.6, COG3315), which plays an important role in the detoxification of catechols in PAH metabolism [18,19], are more abundant in the genomes of PAH-non-degraders (Figure 8), suggesting that some other different functions are probably involved in pathogenicity in the pathogenic mycobacteria (Figure 8). Most enzymes belonging to
SCP, including the decarboxylase, are expressed constitutively, which suggests that their regulation is more relaxed than those belonging to the RCP (Figures $5 \mathrm{C}$ and 7B).

The highly conserved catabolic genes responsible for RCP and SCP in the PAH-degraders clearly indicate that all six PAH-degraders should convert a broad range of PAHs into phthalate, which is the main hub node with an in-degree of 7 and out-degree of 3 in the PAH-MN from $M$. vanbaalenii PYR-1 [9]. In addition, a synteny block for the $\beta$-ketoadipate pathway in the PAH-degraders is conserved (Figure 10B), which further suggests that all outgoing metabolic routes from phthalate could be channeled into the $\beta$-ketoadipate pathway via protocatechuate. Hence, the pca gene cluster for the $\beta$-ketoadipate pathway inevitably functions for CAP in mycobacterial PAH metabolism (Figures 5C, 7B, 8, and 10B).

A closer examination of the pca clusters from the members of PAH-degraders and PAH-non-degraders provides additional evidence not only for a pivotal role of HGT but also for evolutionary dynamics of operon structures in the evolution of the PAH-degrading phenotype at the genus level (Figure 10B). Despite the benefits 


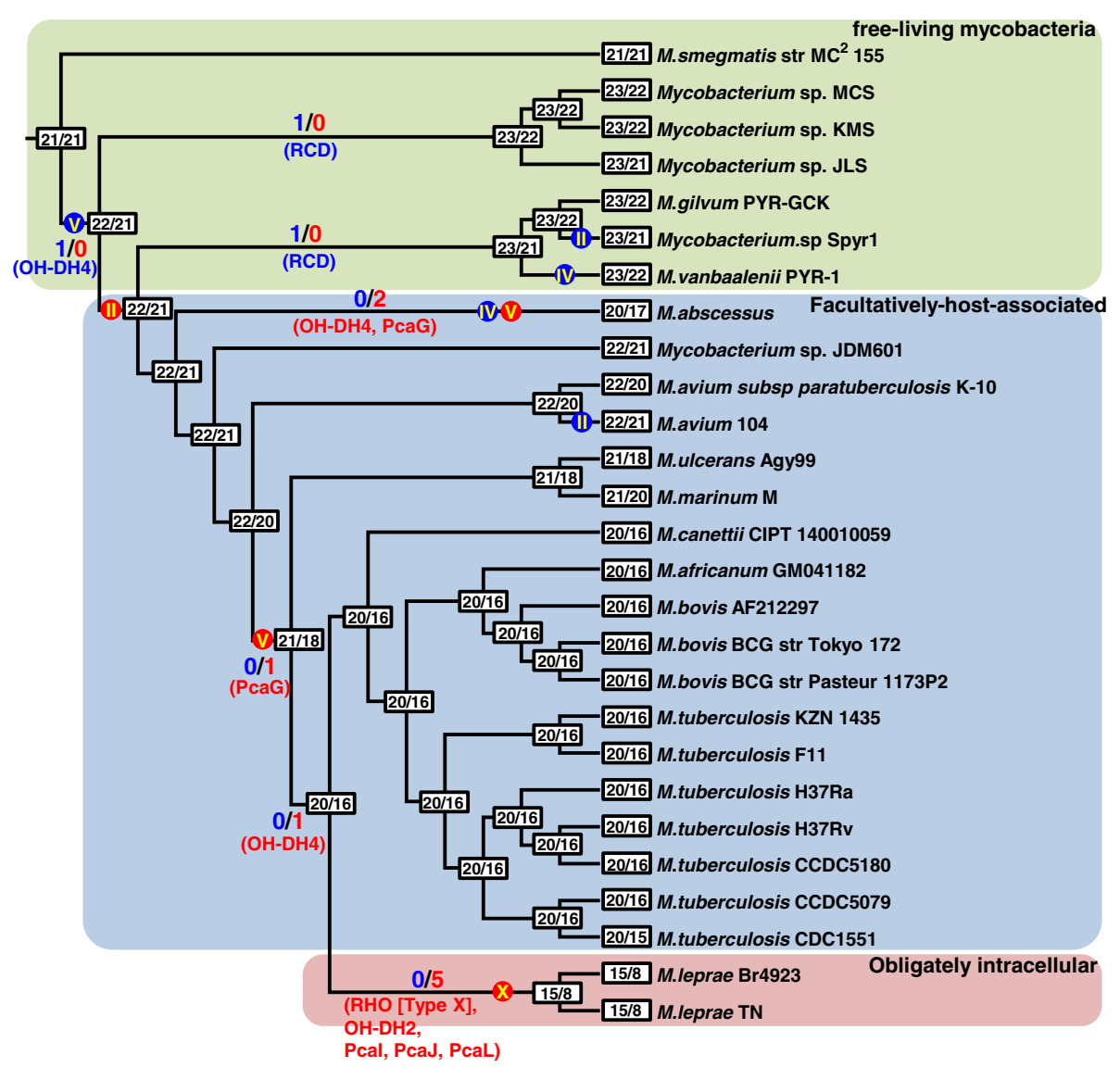

Figure 11 Gain and loss of PAH-degrading genes in the genus Mycobacterium. Boxes on nodes and tips for the phylogeny show numbers of the PAH-degrading gene groups and numbers of the groups with more than one member at least, respectively. Numbers and dots on branches indicate the gains and losses of twenty three PAH-degrading gene groups and four types of RHO groups, respectively, with blue indicating an overall gain and red an overall loss. Roman numerals in the dots indicate type (types II, IV, V and X) of the RHO systems based on Kweon's classification [67,69]. The 27 mycobacteria are color shaded according to their lifestyle: green, free-living; blue, facultatively-host-associated; red, obligately intracellular. $\mathrm{OH}-\mathrm{DH} 4$, alcohol dehydrogenase 4; RCD, ring-cleavage dioxygenase; $\mathrm{PcaG}$, the alpha subunit of protocatechuate 3,4-dioxygenase; $\mathrm{RHO}$, ring-hydroxylating oxygenase; $\mathrm{OH}-\mathrm{DH} 2$, alcohol dehydrogenase 2; Pcal, 3-oxoadipate CoA-transferase subunit A; PcaJ, 3-oxoadipate CoA-transferase subunit B; PcaL, 3-oxoadipate enol-lactone hydrolase/4-carboxymuconolactone decarboxylase.

conferred by coregulation [74], the pca operon is not stable in composition over evolutionary time, as shown in Figure 10B. The pcaHGBLIJ genes seem to be resistant for operon breakage in all PAH-degraders, although the gene $p c a F$, encoding $\beta$-ketoadipyl-CoA thiolase, the last step enzyme to transform $\beta$-ketoadipyl-CoA to succinyl-CoA and acetyl-CoA in the $\beta$-ketoadipate pathway $[9,75]$, does not reside in the pca cluster. In the PAH-non-degraders Mycobacterium sp. JDM601, M. avium 104, and M. avium subsp. paratuberculosis K-10, these operons are drastically reduced, having lost several genes, such as pcaL, pcaI, pcaJ, and pcaF (Figure 10B). However, although several pca genes are found in the genomes of other PAH-non-degraders, they are completely dispersed (Figures 8 and 10B). Especially, the two OI strains Br4923 and TN have completely lost pcaI, $p c a J$, and pcaL (Figure 11). Therefore, stability of the pca operon structure is strongly correlated with the PAH- degrading ability of the genome, functioning as a useful indicator in the assessment of genome evolution in terms of the PAH-degrading phenotype.

\section{A possible evolutionary path of the PAH-degrading phenotype in the genus Mycobacterium}

An integrated view of phylogeny, functional pan-genome, and MPN suggests that mycobacterial genomic dynamics has been communicated to the PAH-degrading phenotype according to its lifestyle. In the genus Mycobacterium, PAH-metabolism might be an attractive backup phenotype for alternative carbon-energy sources to free-living mycobacteria, but it seems to have been regarded as dispensable by the host-dependent species. In addition, due to PAH metabolic ambiguity (as nutrient or toxicant), its evolution might not have been simple or even random. The observed gene gain/loss patterns by HGT and deletion hypothesize a possible evolutionary path in which mycobacteria have 
obtained and preserved the extra metabolic capability while free-living, but during evolution towards the hostdependent life lost this dispensable capability (Figure 12).

\section{Epistasis-based origin of the mycobacterial PAH- degrading phenotype}

Genomic evidence from the 27 fully-sequenced mycobacteria strongly suggests extensive HGT events of the genetic materials related to PAH metabolism in the environment. Furthermore, the genus Mycobacterium originally had several metabolic and physiological selective advantages along with this new metabolic phenotype: (i) high specific affinity for PAH substrates, (ii) cell surface hydrophobicity and attachment ability, and (iii) formation of biofilms on PAH particles $[4,5,14,15]$. Despite such selective advantages, however, the energy required to acquire a PAH-degrading phenotype seems excessively high. For example, for the four ring-structure pyrene to be completely degraded in the PAH-MN, the genomic HGT evidence in this study and the previously proposed evolution hypothesis of the metabolic network suggest that at least 5 different HGT events are required, which should successfully provide the building blocks for a patchwork assembly to cover the entire 27 metabolic steps [6,7]. Actually, since the HGT events can in principle occur in any order, there are $5 !=120$ HGT trajectories linking the gene clusters. However, toxic effects of the uncontrolled intermediates, such as o-quinones [18], might not regard all trajectories equivalently. Therefore, productive epistatic interactions of the building blocks should have a strong selection impact on the evolutionary trajectories. Consistent with this opinion, the conserved genetic sources with similar gene cluster structures in the core-genome of the PAHdegrading mycobacteria suggest overlapping evolutionary events and evolutionary trajectories for the PAH-degrading phenotype. Under strong natural selection towards minimized side effects, impacts of the epistatic interaction of the genetic sources might restrict the possible evolutionary trajectories for mycobacteria to be able to obtain the PAH metabolic ability. Hence, all the successful evolutionary trajectories were able to satisfy the functionally reversed growth of the PAH-MN: a patchwork assembly with a growth direction from CAP to RCP via $\mathrm{SCP}$, essential to minimize the toxic effects of the activated PAH intermediates, which are produced in the RCP [9] (Figures 11 and 12).

\section{Pleiotropy-dependent abandonment of the mycobacterial PAH-degrading phenotype}

Despite a high degree of technical difficulty in obtaining a PAH-degrading ability, it could be an attractive phenotype for free-living mycobacteria in terms of nutritional competition in oligotrophic environmental niches [5,20]. On the other hand, the PAH-degrading phenotype might be unnecessary to host-dependent mycobacteria, which do not obtain nutritional benefits from PAH substrates. For example, M. tuberculosis, an intracellular pathogen, uses fatty acids and cholesterol from its host [76]. Interestingly, as revealed in the gene gain/loss analysis (Figure 11), there is a clear negative correlation between

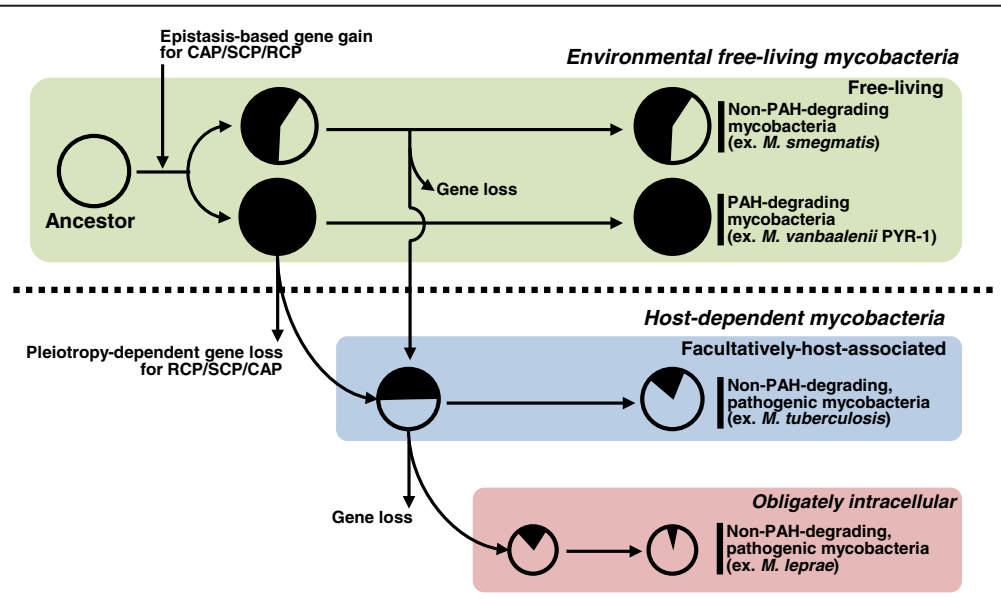

Figure 12 A plausible evolutionary pathway of the PAH-degrading mycobacterial group. The scheme is based on the gene gain and loss of PAH-degrading genes and the genomic information (genome and pan-genomes). The size of circles indicates the ratio of the relative size of the genomes. The complete black circles indicate a mycobacterium with the complete gene set for PAH-degradation, while partially blacked circles indicate PAH-non-degrading mycobacteria with incomplete PAH-degrading genes. More filled circles mean more PAH-degrading genes in the genome. Color shading follows Figure 11. Note that all PAH-degrading mycobacteria have larger genome size and more HGT events than PAH-non-degrading mycobacteria, which have some PAH-degrading genes but not a complete set of genes. Especially, Ol mycobacteria have the smallest size in terms of genome and the number of PAH-degrading genes. Under strong natural selection, the gene gain/loss by HGT/deletion events hypothesize an epistasis-based birth (from CAP to RCP via SCP) and pleiotropy-dependent death (from RCP and CAP to SCP), for PAH metabolism in the genus Mycobacterium. 
the size of genetic remnants related to PAH metabolism and the evolutionary time toward the host-dependent life, which suggests the basic evolutionary concept-initially adopted but later abandoned-for the PAH-degrading phenotype in the genus Mycobacterium (Figure 12). The instability of the operon structures remaining in the genomes of the host-dependent mycobacteria and the lack of certain genes for PAH catabolic function suggest abandonment of the PAH-degrading phenotype in the evolution towards a host-dependent lifestyle. Because PAHs are absent in the biological hosts, it is likely that this ability has been lost during the host-dependent evolution.

We observed the early events in the disassembly of the PAH-MN in the FHA PAH-non-degrading mycobacterial genomes (Mycobacterium sp. JDM601, M. avium 104, and $M$. avium subsp. paratuberculosis K-10), which are located in the middle of the free-living PAH-degraders and the FHA M. tuberculosis complex, while there was a rare opportunity to observe the genetic evidence from the free-living PAH-degrading mycobacteria (Figures 10 and 11). As shown in Figure 10, the operons having genes involved in the phthalate and $\beta$-ketoadipate pathways, respectively, according to the PAH-metabolic ability showed different stability; the two operons are protected from breakage in the PAH-degrading strains but are broken in the PAH-non-degrading mycobacterial genomes.

Like the origin of PAH-MN discussed above, the same strength of natural selection minimizing toxic side effects might also have restricted the abandonment trajectories in the evolutionary process for disuse of the dispensable metabolic capability in the host-dependent mycobacteria. Evolutionary rational endeavors seem to have participated in the disassembly process to follow the effective abandonable trajectories to minimize any possible side-effects and to save time and energy. In this respect, the effects of genetic perturbation on functional robustness-correlation between functional redundancy and negative epistasis-from the perspective of quantity and quality in the metabolism of PAHs provide clues to understand the plausible abandonment trajectories. Although the PAHdegrading phenotype is functionally robust to genetic perturbation, the robustness depends mainly on perturbation location in the PAH-MN. The funnel-like topology of the $\mathrm{MN}$ is intimately related to its behavior and also to its functional robustness toward genetic perturbation, in which many peripheral pathways converge to the $\beta$ ketoadipate pathway with the same input and output diameters in its function, which is linked to the TCA cycle [9]. In general, effects of the functional perturbation on the steps, with extremely low genetic and functional redundancy, propagate across the entire PAH-MN, creating many possible quantitative metabolic damages. In this respect, as revealed in the pan-genomic and RHO profile comparison, the host-dependent mycobacteria seem to have followed the effective abandonment trajectories, which initially tried to have the RHO systems disabled from the RCP modules, responsible mainly for toxic PAH intermediates. However, from the negative epistatic interaction view, $\mathrm{RHO}$ systems in the PAH-MN are not good target genes for effective abandonment trajectories, considering their extremely high genetic and functional redundancy. Surprisingly, the host-dependent mycobacteria seem to find the best solution for this problematic requirement from the common feature of mycobacterial RHO systems, which is numerical imbalance between the two functional groups, an oxygenase and an ETC $[9,67,68]$. As shown in Figure 13, the distinct numerical imbalance between oxygenase components and an ETC clearly indicates that a gene encoding the ETC has strong functionally pleiotropic effects. Based on these

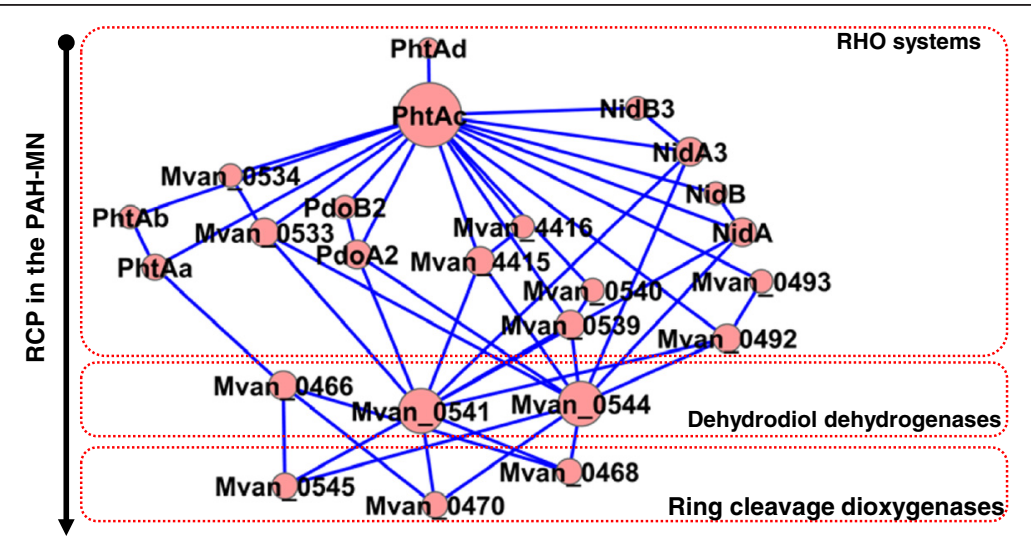

Figure 13 Scale-free functional interaction network of the enzymes responsible for RCP in the PAH-MN. The functional network was reconstructed based on the enzymes involved in the RCP in PAH-MN, which are expressed as proteins and exist in the core-genome of the PAH-degraders (that is, selected by functional pan-genomics). In the network, the size of nodes is based on the degree of connectivity of the nodes. The main hub node, PhtAc, which shows the highest connectivity with an in-degree of 1 and out-degree of 16, is shown as the biggest node in size. Cytoscape was used for visualizing and analyzing networks in this study [77]. 
observations, the practical metabolic effects of the gene loss for a type V ETC could be significant and effective. Especially, considering their responsibility for the initial ring-hydroxylation of PAH substrates in diverse RCP functional modules (Figure 13), which produce activated toxic intermediates, removal of the RHO function by loss of the strong pleiotropic gene could be effective and time-saving, as observed in the metabolic and proteomic data of PAH-MN [9]. In addition, the three type $\mathrm{V}$ RHO enzymes, two Nid systems and a Pdo system responsible mainly for the initial oxygenation of diverse $\mathrm{PAH}$ substrates are absent in all PAH-non-degraders. Together with these endeavors, the PAH-non-degraders have kept the enzymes responsible for detoxification. For example, more genes encoding COMTs, involved in the detoxification of toxic PAH intermediates, exist in the PAH-non-degraders (Figure 8).

Taken together, these systematic observations offer strong support for the hypothesis that natural selection for maximized metabolic benefits and minimized side effects of the uncontrolled PAH intermediates has restricted the evolutionary trajectories of mycobacterial PAH metabolism. The evolutionary behavior might take full advantage of the pleiotropic and epistatic effects of gene gain/loss, allowing mycobacteria to follow the few successful trajectories, which satisfy the functional and toxicological requirements in the evolution of PAH metabolism in the genus Mycobacterium.

\section{Conclusions}

Taking the whole picture into account, mycobacterial PAH metabolism at the genus level might be an excellent phenotypic model to bridge genomic dynamics and phenotypic evolution, which could address a series of questions for a predictable direction and plausible trajectories for mycobacterial evolution. The reasonable factors and their practical advantages include the following: (i) the metabolic ambiguity of PAHs as carbon and energy sources or toxicants, which might have directed evolutionary responses to maximize the nutritional benefits and minimize the possible side-effects, enhancing opportunities to observe the apparently selective choice of evolutionary trajectories; (ii) the availability (ubiquity) of PAHs in the environment but not in the biological hosts, which might be an apparent selective pressure on its evolution; (iii) the flood of genome sequences and phenotypic information across strains from free-living to obligate intracellular parasites, together with the complete metabolic and genetic knowledge for mycobacterial $\mathrm{PAH}$ metabolism, which allow synergistic analysis of phylogenic, phenotypic, and genomic information going into the structure, behavior, and evolution of PAH metabolism.

In this study, we have discovered a wealth of evidence relating mycobacterial evolution to $\mathrm{PAH}$ metabolism. This evidence comes in a useful mycobacterial compendium of phenotypic and genomic changes, focusing on the $\mathrm{PAH}$-degrading phenotype with a functional pan-genomic perspective of the evolutionary events and environmental challenges. The compendium enabled us to understand the relation between genomic dynamics and the corresponding phenotypic effects, which helped us to follow a few plausible evolutionary paths to fitter phenotypes. When selection acts on evolution of PAH metabolism, only a small fraction of evolutionary trajectories are likely to be observed, owing mainly to a combination of the ambiguous metabolic effects of PAHs and the corresponding pleiotropy- and epistasis-dependent evolutionary behavior. Systematic functional pan-genomic comparison allows us to follow largely identical evolutionary trajectories, which substantially enriches our understanding of the pleiotropic and epistatic evolutionary process of $\mathrm{PAH}$ metabolism at the genus level. Evolutionary constraints on the trajectories seen in the PAH-degrading phenotype are likely to apply to the evolution of other phenotypes in the genus Mycobacterium, which is essential to better characterize mycobacterial communities for medical or bioengineering applications.

\section{Methods}

\section{Genome sequences and proteomic data sets}

In this study, we used only complete genome sequences with well-organized phenotypic and genomic information (Table 1) for reasonable network and functional pangenomic analysis without numerical bias in terms of mycobacterial phenotypes. All genome sequences were retrieved from the NCBI (http://www.ncbi.nlm.nih.gov/ genome/browse/) or IMG/JGI (https://img.jgi.doe.gov/ cgi-bin/w/main.cgi). The NCBI gbk and ptt (a tab delimited file containing a list of all the proteins for the genome) files of the 27 genome sequences were obtained from the NCBI ftp site (ftp://ftp.ncbi.nih.gov/genomes/) to generate a master genome table for gene annotation and COG analysis of the pan-genome and proteomic data sets. For functional pan-genomic comparison, the proteomic data sets of $M$. vanbaalenii PYR-1 (DSM 7251) treated with phthalate, the LMW PAHs fluorene, acenaphthylene, anthracene, and phenanthrene, and the HMW PAHs pyrene and benzo[a]pyrene, were used to select the genes which are expressed as proteins from the pan-genomic data $[6-9,67,68]$.

\section{Genome comparison}

Basic comparative genome analyses, including genome clustering based on COG information, were conducted using the JGI Integrated Microbial Genomes (IMG) system (https://img.jgi.doe.gov/cgi-bin/w/main.cgi). To visualize the function-based genomic comparison, the 27 mycobacterial genomes were clustered based on functional 
profiles; the PCA clustering analysis was conducted using the genome clustering function of the JGI IMG system (https://img.jgi.doe.gov/cgi-bin/w/main.cgi) with the condition: clustering type, COG and clustering method, Principal Components Analysis (PCA). Proximity of grouping indicates the similarity of genomes.

Pan-genome (core and pan-genome) and Venn diagram analyses of the pan-genomic data sets were conducted using EDGAR [78]. The core and pan-genome were computed by iterative pairwise comparison of a set of genomes. Using the metacontig function of EDGAR, we also defined custom groups of genomes for which the core genome or the pan genome have been stored as virtual contigs. A total of 9 virtual contigs according to the phenotype grouping of the 27 mycobacterial genomes was used in all subsequent EDGAR calculations, including Venn diagram analysis.

\section{Phylogenetic analysis}

For a concatenated phylogenetic tree, we selected the 22 common genes from the core genome in the 27 mycobacterial strains [79] and estimated phylogenetic relationships. The 22 genes include (locus tags from $M$. vanbaalenii PYR-1); Mvan_0002, Mvan_1234, Mvan_1235, Mvan_1236, Mvan_1258, Mvan_1280, Mvan_1282, Mvan_1305, Mvan_1308, Mvan_1311, Mvan_1312, Mvan_1334, Mvan_1337, Mvan_1339, Mvan_1347, Mvan_1434, Mvan_1435, Mvan_1436, Mvan_1470, Mvan_1489, Mvan_2209, and Mvan_2651. Phylogenetic trees were constructed by the maximum likelihood method using PhyML [80] with the following parameters: (1) input data: the concatenated DNA sequences of the 22 common genes; (2) substitution model: HKY85 with substitution rate categories of 4 and estimated gamma shape parameter; (3) tree searching: BIONJ for starting tree and NINI for tree improvement with optimizing of topology and branch lengths; and (4) bootstrap: 100. MEGA4 [81] was used to visualize and manipulate the output trees.

\section{Gain/loss analysis of the PAH-degrading genes}

The gain and loss of the PAH-degradation gene groups (a total of 23 groups) was assessed using the Count software [72] based on the PAH-degrading genes matrix across the genus Mycobacterium (Additional file 4: Table S13 and Additional file 5: Table S14). The Wagner parsimony model implemented in Count with the gain penalty of 1 was used to reconstruct gene content evolution in the history of the PAH-degrading gene groups as well as four types of the RHO group. The phylogenetic tree constructed by the maximum likelihood method (Figure 2A) was used as the guide topology.

\section{Ring hydroxylating oxygenase ( $\mathrm{RHO}$ ) classification}

For classification and comparison of mycobacterial RHO enzymes, we used ClassRHO [67-69], which performs hierarchical cluster analysis using pairwise distance (PD) matrices and calculated from ClustalW default protein weight matrix (Blosum). For the classification of 246 RHO systems from the 27 mycobacterial strains, we used the automatic optimal threshold value that maximizes the learning accuracy. In the analysis, together with 42 standard RHO sequences, the amino acid sequences of large $(\alpha)$ subunits from the $246 \mathrm{RHO}$ systems were used as RHO query sequences.

\section{Genomic island (GI) analysis}

For computational identification, comparison, and visualization of genomic islands from the mycobacterial genomes, we used IslandViewer [82], a computational tool which integrates three different genomic island prediction methods, IslandPick [82], SIGI-HMM [83], and IslandPath-DIMOB [84].

\section{Network model of the relationships of the mycobacterial phenotypes}

To model relationships and strengths among the nine phenotypes (Free-Living, PAH-degrading, Fast-growing, Non-pathogenic, Facultatively-host-associated, PAH-nondegrading, Pathogenic, Slow-growing, and Obligately intracellular), we reconstructed a network, Mycobacterial Phenotype Network (MPN). Basically, the network consists of nodes (vertices) and connections (edges) to represent mycobacteria and their relations, respectively. Since the phenotypes seem to be disjoint or individual sets, whose nodes (i.e. mycobacteria) can be partitioned into subsets, the structure between two groups (phenotypes) is loosely based on a bipartite graph. Seemingly, in the MPN several bipartite graphs are interrelated; however, different phenotypes may have identical mycobacteria, which are represented by individual nodes in each phenotype, to model the relationships. To represent the relationships, we connect the nodes between two phenotypes only if they are identical mycobacteria, i.e. the same mycobacterial occurrence/event between two phenotypes. In the MPN, the connection is undirected since it includes no distinction or causality between the nodes. Intuitively, in the MPN, the greater the number of edges between groups, the stronger the connection is. We define the connection strength between two groups, Gx and Gy, as CS (Gx, Gy), defined as

$$
C S(G x, G y)=[\rho(G x)+\rho(G y)] * 50
$$

where $\rho(G x)$ is the percentage of nodes in Gx with edges to Gy. 
For visualizing and analyzing networks in this study, Cytoscape [77] was used. In the MPN, the thickness of edge between two phenotype groups is based on the degree of the connection strength (CS value).

\section{Availability of supporting data}

The data sets supporting the phylogenetic result of this article are available on the TreeBASE repository, ID: S16971, http://purl.org/phylo/treebase/phylows/study/TB2:S16971.

\section{Additional files}

Additional file 1: Table S1. Core-genome analysis of the six PAH-degrading mycobacteria. Table S2. Functional analysis of the 3,533-gene coregenome. Table S3. Analysis of 136 genes involved in PAH metabolism. Table S4. Protein expression profile of 136 genes in M. vanbaalenii PYR-1. Table S5. List of 113 genes out of the 136 genes, which are expressed as proteins involved in PAH degradation pathways.

Additional file 2: Table S6. Analysis of HGT in the six PAH-degrading mycobacteria. Table S7. List of 754 proteins contributing to the core-genome from a total of 3,533 genes. Table S8. List of 2,124 proteins contributing to the dispensable genome from a total of 5,999 genes. Table S9. List of 29 proteins, based on COG categories, responsible for all enzymatic reactions involved in the degradation of PAHs in M. vanbaalenii PYR-1. Table S10. List of 29 proteins, based on functional modules, responsible for all enzymatic reactions involved in the degradation of PAHs in $M$. vanbaalenii PYR-1.

Additional file 3: Table S11. List of 248 core genes involved in PAH degradation identified in M. vanbaalenii PYR-1. Table S12. Summary of 248 core genes.

Additional file 4: Table S13. Distribution of PAH-degrading genes across the genus Mycobacterium.

Additional file 5: Table S14. Distribution of RHO enzymes across the genus Mycobacterium.

\section{Abbreviations}

PAHs: Polycyclic aromatic hydrocarbons; HMW: High-molecular-weight; LMW: Low-molecular-weight; HGT: Horizontal gene transfer; PAH-MN: PAH Metabolic network; MPN: Mycobacterial phenotype network; FL: Free-living; FHA: Facultatively-host-associated; Ol: Obligately intracellular; $\mathrm{RHO}$ : Ring-hydroxylating oxygenase; ETC: Electron transfer component; PN: Phenotype network; COG: Cluster of orthologous groups; CYP: Cytochrome P450 monooxygenase; RCD: Ring-cleavage dioxygenase; PCA: Principal components analysis; PD: Pairwise distance; NCBI: National Center for Biotechnology Information; IMG: Integrated Microbial Genomes; JGI: Joint Genome Institute.

\section{Competing interests}

The authors declare that they have no competing interests.

\section{Authors' contributions}

OK, SK, JBS, and CEC conceived the project, designed research, performed research, analyzed data, and wrote the manuscript; JB designed research (Pan-genome analysis) and wrote the manuscript; SKK, BSK, and DHB analyzed data (database and phylogeny) and wrote the manuscript. All authors read and approved the final manuscript.

\section{Acknowledgements}

We thank Kuppan Gokulan and Mark Hart for critical review of the manuscript. We also thank Dr. Joshua Xu for his statistical advice. This work was supported in part by an appointment to the Postgraduate Research Fellowship Program at the National Center for Toxicological Research, administered by the Oak Ridge Institute for Science and Education through an interagency agreement between the U. S. Department of Energy and the U. S. Food and Drug Administration. The views presented in this article do not necessarily reflect those of the U.S. FDA.

\section{Author details}

'Division of Microbiology, National Center for Toxicological Research/FDA, Jefferson, Arkansas, USA. ${ }^{2}$ Center for Biotechnology, Bielefeld University, Bielefeld, Nordrhein-Westfalen, Germany. ${ }^{3}$ Department of Management, University of Arkansas at Little Rock, Little Rock, Arkansas, USA. ${ }^{4}$ Department of Life Science, Hallym University, Chuncheon, Gangwon-do 200-702, Republic of Korea. ${ }^{5}$ Department of Oral Microbiology and Immunology, School of Dentistry, Dankook University, Chonan, Republic of Korea.

${ }^{6}$ Department of Computer Science and Engineering, Texas A\&M University, College Station, Texas, USA.

Received: 19 September 2014 Accepted: 29 January 2015 Published online: 14 February 2015

\section{References}

1. Rastogi N, Legrand E, Sola C. The mycobacteria: an introduction to nomenclature and pathogenesis. Rev Sci Tech. 2001;20:21-54.

2. Hartmans S, De Bont JA, Stackebrandt E. The genus Mycobacterium-nonmedical. 3rd ed. New York, NY: Springer Verlag; 2006.

3. Saviola B, Bishai W. The genus Mycobacterium-medical. In: Falkow S, Rosenberg E, Schleifer K-H, Stackebrandt E, editors. The Prokaryotes. 3rd ed. New York, NY: Springer Verlag; 2006. p. 919-33.

4. Glickman MS, Jacobs Jr WR. Microbial pathogenesis of Mycobacterium tuberculosis: dawn of a discipline. Cell. 2001;104:477-85.

5. Kim SJ, Kweon O, Cerniglia CE. Degradation of polycyclic aromatic hydrocarbons by Mycobacterium strains. In: Timmis KN, editor. Handbook of hydrocarbon and lipid microbiology, vol. 3. Braunschweig, Germany: Springer; 2010. p. 1865-80 [McGenity T (Series Editor): Microbes and Communities Utilizing Hydrocarbons, Oils and Lipids].

6. Kim SJ, Kweon O, Jones RC, Freeman JP, Edmondson RD, Cerniglia CE. Complete and integrated pyrene degradation pathway in Mycobacterium vanbaalenii PYR-1 based on systems biology. J Bacteriol. 2007;189:464-72.

7. Kweon O, Kim SJ, Jones RC, Freeman JP, Adjei MD, Edmondson RD, et al. A polyomic approach to elucidate the fluoranthene degradative pathway in Mycobacterium vanbaalenii PYR-1. J Bacteriol. 2007;189:4635-47.

8. Kim SJ, Song J, Kweon O, Holland RD, Kim DW, Kim JN, et al. Functional robustness of a polycyclic aromatic hydrocarbon metabolic network examined in a nidA aromatic ring hydroxylating oxygenase mutant of Mycobacterium vanbaalenii PYR-1. Appl Environ Microbiol. 2012;78:3715-23.

9. Kweon O, Kim SJ, Holland RD, Chen H, Kim DW, Gao Y, et al. Polycyclic aromatic hydrocarbon-metabolic network in Mycobacterium vanbaalenii PYR-1. J Bacteriol. 2011;193:4326-37.

10. DeBruyn JM, Mead TJ, Sayler GS. Horizontal transfer of PAH catabolism genes in Mycobacterium: evidence from comparative genomics and isolated pyrene-degrading bacteria. Environ Sci Technol. 2011;46:99-106.

11. Badejo AC, Choi CW, Badejo AO, Shin KH, Hyun JH, Lee YG, et al. A global proteome study of Mycobacterium gilvum PYR-GCK grown on pyrene and glucose reveals the activation of glyoxylate, shikimate and gluconeogenetic pathways through the central carbon metabolism highway. Biodegradation. 2013;24:741-52.

12. Miller CD, Hall K, Liang YN, Nieman K, Sorensen D, Issa B, et al. Isolation and characterization of polycyclic aromatic hydrocarbon-degrading Mycobacterium isolates from soil. Microb Ecol. 2004;48:230-8.

13. Kallimanis A, Karabika E, Mavromatis K, Lapidus A, Labutti KM, Liolios K, et al. Complete genome sequence of Mycobacterium sp. strain (Spyr1) and reclassification to Mycobacterium gilvum Spyr1. Stand Genomic Sci. 2011;5:144-53.

14. Kweon O, Kim SJ, Cerniglia CE. Genomic view of mycobacterial high molecular weight polycyclic aromatic hydrocarbon degradation. In: Timmis KN, editor. Handbook of hydrocarbon and lipid microbiology, vol. 2. Braunschweig, Germany: Springer; 2010. p. 1165-78.

15. Kweon O, Kim SJ, Freeman JP, Song J, Baek S, Cerniglia CE. Substrate specificity and structural characteristics of the novel Rieske nonheme iron aromatic ring-hydroxylating oxygenases NidAB and NidA3B3 from Mycobacterium vanbaalenii PYR-1. MBio. 2010;1:e00135-10.

16. Ostman B, Hintze A, Adami C. Impact of epistasis and pleiotropy on evolutionary adaptation. Proc Biol Sci. 2012;279:247-56.

17. Heitkamp MA, Freeman JP, Miller DW, Cerniglia CE. Pyrene degradation by a Mycobacterium sp.: identification of ring oxidation and ring fission products. Appl Environ Microbiol. 1988;54:2556-65. 
18. Kim YH, Moody JD, Freeman JP, Brezna B, Engesser KH, Cerniglia CE. Evidence for the existence of PAH-quinone reductase and catechol-Omethyltransferase in Mycobacterium vanbaalenii PYR-1. J Ind Microbiol Biotechnol. 2004;31:507-16.

19. Kim SJ, Jones RC, Cha CJ, Kweon O, Edmondson RD, Cerniglia CE. Identification of proteins induced by polycyclic aromatic hydrocarbon in Mycobacterium vanbaalenii PYR-1 using two-dimensional polyacrylamide gel electrophoresis and de novo sequencing methods. Proteomics. 2004:4:3899-908

20. Cerniglia CE. Biodegradation of polycyclic aromatic hydrocarbons. Biodegradation. 1992;3:351-68.

21. Kanaly RA, Harayama S. Advances in the field of high-molecular-weight polycyclic aromatic hydrocarbon biodegradation by bacteria. Microb Biotechnol. 2011;3:136-64.

22. Cheung PY, Kinkle BK. Mycobacterium diversity and pyrene mineralization in petroleum-contaminated soils. Appl Environ Microbiol. 2001;67:2222-9.

23. Tyler AL, Asselbergs FW, Williams SM, Moore JH. Shadows of complexity: what biological networks reveal about epistasis and pleiotropy. Bioessays. 2009;31:220-7

24. Zakham F, Aouane O, Ussery D, Benjouad A, Ennaji MM. Computational genomics-proteomics and Phylogeny analysis of twenty one mycobacterial genomes (Tuberculosis \& non Tuberculosis strains). Microb Inform Exp. 2012:2:7.

25. Jena L, Kumar S, Harinath BC. MycoProtease-DB: useful resource for Mycobacterium tuberculosis complex and nontuberculous mycobacterial proteases. Bioinformation. 2012;8:1240-2.

26. Kim YH, Engesser $\mathrm{KH}$, Cerniglia CE. Numerical and genetic analysis of polycyclic aromatic hydrocarbon-degrading mycobacteria. Microbial Ecol. 2005:50:110-9.

27. Khan AA, Kim SJ, Paine DD, Cerniglia CE. Classification of a polycyclic aromatic hydrocarbon-metabolizing bacterium, Mycobacterium sp. strain PYR-1, as Mycobacterium vanbaalenii sp. nov. Int J Syst Evol Microbiol. 2002:52:1997-2002

28. Dean-Ross D, Cerniglia CE. Degradation of pyrene by Mycobacterium flavescens. Appl Microbiol Biotechnol. 1996;46:307-12.

29. Zakham F, Belayachi L, Ussery D, Akrim M, Benjouad A, El Aouad R, et al. Mycobacterial species as case-study of comparative genome analysis. Cell Mol Biol (Noisy-le-Grand). 2011;57(Suppl):OL1462-9.

30. Zhang ZY, Sun ZQ, Wang ZL, Hu HR, Wen ZL, Song YZ, et al. Identification and pathogenicity analysis of a novel non-tuberculous Mycobacterium clinical isolate with nine-antibiotic resistance. Clin Microbiol Infect. 2013;19:91-6.

31. Stinear TP, Seemann T, Harrison PF, Jenkin GA, Davies JK, Johnson PD, et al. Insights from the complete genome sequence of Mycobacterium marinum on the evolution of Mycobacterium tuberculosis. Genome Res. 2008;18:729-41.

32. Stinear TP, Seemann T, Pidot S, Frigui W, Reysset G, Garnier T, et al. Reductive evolution and niche adaptation inferred from the genome of Mycobacterium ulcerans, the causative agent of Buruli ulcer. Genome Res. 2007:17:192-200.

33. Stinear TP, Mve-Obiang A, Small PL, Frigui W, Pryor MJ, Brosch R, et al. Giant plasmid-encoded polyketide synthases produce the macrolide toxin of Mycobacterium ulcerans. Proc Natl Acad Sci U S A. 2004;101:1345-9.

34. Torrelles JB, Ellis D, Osborne T, Hoefer A, Orme IM, Chatterjee D, et al. Characterization of virulence, colony morphotype and the glycopeptidolipid of Mycobacterium avium strain 104. Tuberculosis (Edinb). 2002;82:293-300.

35. Horan KL, Freeman R, Weigel K, Semret M, Pfaller S, Covert TC, et al. Isolation of the genome sequence strain Mycobacterium avium 104 from multiple patients over a 17-year period. J Clin Microbiol. 2006;44:783-9.

36. Ripoll F, Pasek S, Schenowitz C, Dossat C, Barbe V, Rottman M, et al. Non mycobacterial virulence genes in the genome of the emerging pathogen Mycobacterium abscessus. PLoS One. 2009;4:e5660.

37. Geluk A, Klein MR, Franken KL, van Meijgaarden KE, Wieles B, Pereira KC, et al. Postgenomic approach to identify novel Mycobacterium leprae antigens with potential to improve immunodiagnosis of infection. Infect Immun. 2005;73:5636-44

38. Li L, Bannantine JP, Zhang Q, Amonsin A, May BJ, Alt D, et al. The complete genome sequence of Mycobacterium avium subspecies paratuberculosis. Proc Natl Acad Sci U S A. 2005;102:12344-9.

39. Paustian ML, Zhu X, Sreevatsan S, Robbe-Austerman S, Kapur V, Bannantine JP. Comparative genomic analysis of Mycobacterium avium subspecies obtained from multiple host species. BMC Genomics. 2008;9:135.

40. Bentley SD, Comas I, Bryant JM, Walker D, Smith NH, Harris SR, et al. The genome of Mycobacterium africanum West African 2 reveals a lineage-specific locus and genome erosion common to the M. tuberculosis complex. PLoS Negl Trop Dis. 2012;6:e1552

41. Hosen MI, Tanmoy AM, Mahbuba DA, Salma U, Nazim M, Islam MT, et al. Application of a subtractive genomics approach for in silico identification and characterization of novel drug targets in Mycobacterium tuberculosis F11. Interdiscip Sci. 2014;6:48-56.

42. Philipp WJ, Nair S, Guglielmi G, Lagranderie M, Gicquel B, Cole ST. Physical mapping of Mycobacterium bovis BCG Pasteur reveals differences from the genome map of Mycobacterium tuberculosis H37Rv and from M. bovis. Microbiology. 1996;142:3135-45.

43. Philipp WJ, Poulet S, Eiglmeier K, Pascopella L, Balasubramanian V, Heym B, et al. An integrated map of the genome of the tubercle bacillus, Mycobacterium tuberculosis H37Rv, and comparison with Mycobacterium leprae. Proc Natl Acad Sci U S A. 1996:93:3132-7.

44. Cole ST, Barrell BG. Analysis of the genome of Mycobacterium tuberculosis H37Rv. Novartis Found Symp. 1998;217:160-72. discussion 172-167.

45. Jiang Y, Liu HC, Zheng HJ, Tang B, Dou XF, Zhao XQ, et al. Evaluation of four candidate VNTR Loci for genotyping 225 Chinese clinical Mycobacterium tuberculosis complex strains. Biomed Environ Sci. 2012;25:82-90.

46. Lu B, Dong HY, Zhao XQ, Liu ZG, Liu HC, Zhang YY, et al. A new multilocus sequence analysis scheme for Mycobacterium tuberculosis. Biomed Environ Sci. 2012;25:620-9.

47. Agarwal N, Bishai WR. CAMP signaling in Mycobacterium tuberculosis. Indian J Exp Biol. 2009;47:393-400

48. Vishnoi A, Roy R, Bhattacharya A. Comparative analysis of bacterial genomes: identification of divergent regions in mycobacterial strains using an anchorbased approach. Nucleic Acids Res. 2007;35:3654-67.

49. Zhang Y, Chen C, Liu J, Deng H, Pan A, Zhang L, et al. Complete genome sequences of Mycobacterium tuberculosis strains CCDC5079 and CCDC5080, which belong to the Beijing family. J Bacteriol. 2011;193:5591-2.

50. Mostowy S, Onipede A, Gagneux S, Niemann S, Kremer K, Desmond EP, et al. Genomic analysis distinguishes Mycobacterium africanum. J Clin Microbiol. 2004;42:3594-9.

51. Gehre F, Otu J, DeRiemer K, de Sessions PF, Hibberd ML, Mulders W, et al. Deciphering the growth behaviour of Mycobacterium africanum. PLoS Neg Trop Dis. 2013;7:e2220.

52. Seki M, Honda I, Fujita I, Yano I, Yamamoto S, Koyama A. Whole genome sequence analysis of Mycobacterium bovis bacillus Calmette-Guerin (BCG) Tokyo 172: a comparative study of BCG vaccine substrains. Vaccine. 2009:27:1710-6.

53. Garnier T, Eiglmeier K, Camus JC, Medina N, Mansoor H, Pryor M, et al. The complete genome sequence of Mycobacterium bovis. Proc Natl Acad Sci U S A. 2003;100:7877-82.

54. Cole ST, Eiglmeier K, Parkhill J, James KD, Thomson NR, Wheeler PR, et al Massive gene decay in the leprosy bacillus. Nature. 2001;409:1007-11.

55. Eiglmeier K, Honore N, Woods SA, Caudron B, Cole ST. Use of an ordered cosmid library to deduce the genomic organization of Mycobacterium leprae. Mol Microbiol. 1993;7:197-206.

56. Monot M, Honore N, Garnier T, Zidane N, Sherafi D, Paniz-Mondolfi A, et al Comparative genomic and phylogeographic analysis of Mycobacterium leprae. Nat Genet. 2009;41:1282-9.

57. Grange JM. The biology of the genus Mycobacterium. Soc Appl Bacteriol Symp Ser. 1996;25:1S-9S.

58. Macia J, Sole RV, Elena SF. The causes of epistasis in genetic networks. Evolution. 2012;66:586-96.

59. Merhej $\mathrm{V}$, Royer-Carenzi M, Pontarotti P, Raoult D. Massive comparative genomic analysis reveals convergent evolution of specialized bacteria. Biol Direct. 2009;4:13

60. Medini D, Donati C, Tettelin H, Masignani V, Rappuoli R. The microbial pan-genome. Curr Opin Genet Dev. 2005;15:589-94.

61. Sreevatsan S, Pan X, Stockbauer KE, Connell ND, Kreiswirth BN, Whittam TS, et al. Restricted structural gene polymorphism in the Mycobacterium tuberculosis complex indicates evolutionarily recent global dissemination. Proc Natl Acad Sci U S A. 1997;94:9869-74.

62. Brown-Elliott BA, Wallace Jr RJ. Clinical and taxonomic status of pathogenic nonpigmented or late-pigmenting rapidly growing mycobacteria. Clin Microbiol Rev. 2002;15:716-46.

63. Krzywinska E, Krzywinski J, Schorey JS. Naturally occurring horizontal gene transfer and homologous recombination in Mycobacterium. Microbiology. 2004;150:1707-12. 
64. Davids W, Zhang Z. The impact of horizontal gene transfer in shaping operons and protein interaction networks-direct evidence of preferential attachment. BMC Evol Biol. 2008;8:23.

65. de Visser JA, Cooper TF, Elena SF. The causes of epistasis. Proc Biol Sci. 2011;278:3617-24.

66. Kvitek DJ, Sherlock G. Reciprocal sign epistasis between frequently experimentally evolved adaptive mutations causes a rugged fitness landscape. PLoS Genet. 2011;7:e1002056.

67. Kweon O, Kim SJ, Baek S, Chae JC, Adjei MD, Baek DH, et al. A new classification system for bacterial Rieske non-heme iron aromatic ring-hydroxylating oxygenases. BMC Biochem. 2008;9:11.

68. Kim SJ, Kweon O, Jones RC, Edmondson RD, Cerniglia CE. Genomic analysis of polycyclic aromatic hydrocarbon degradation in Mycobacterium vanbaalenii PYR-1. Biodegradation. 2008;19:859-81.

69. Baek S, Kweon O, Kim SJ, Baek DH, Chen JJ, Cerniglia CE. ClassRHO: a platform for classification of bacterial Rieske non-heme iron ring-hydroxylating oxygenases. J Microbiol Methods. 2009:76:307-9.

70. Kim SJ, Kweon O, Freeman JP, Jones RC, Adjei MD, Jhoo JW, et al. Molecular cloning and expression of genes encoding a novel dioxygenase involved in low- and high-molecular-weight polycyclic aromatic hydrocarbon degradation in Mycobacterium vanbaalenii PYR-1. Appl Environ Microbiol. 2006;72:1045-54.

71. Khan AA, Wang RF, Cao WW, Doerge DR, Wennerstrom D, Cerniglia CE. Molecular cloning, nucleotide sequence, and expression of genes encoding a polycyclic aromatic ring dioxygenase from Mycobacterium sp. strain PYR1. Appl Environ Microbiol. 2001;67:3577-85

72. Csuros M. Count: evolutionary analysis of phylogenetic profiles with parsimony and likelihood. Bioinformatics. 2010;26:1910-2.

73. Brezna B, Kweon O, Stingley RL, Freeman JP, Khan AA, Polek B, et al. Molecular characterization of cytochrome P450 genes in the polycyclic aromatic hydrocarbon degrading Mycobacterium vanbaalenii PYR-1. Appl Microbiol Biotechnol. 2006;71:522-32.

74. Price MN, Arkin AP, Alm EJ. The life-cycle of operons. PLoS Genet. 2006;2:e96.

75. Harwood CS, Parales RE. The ß-ketoadipate pathway and the biology of self-identity. Annu Rev Microbiol. 1996;50:553-90.

76. Niederweis M. Nutrient acquisition by mycobacteria. Microbiology. 2008;154:679-92.

77. Shannon P, Markiel A, Ozier O, Baliga NS, Wang JT, Ramage D, et al. Cytoscape: a software environment for integrated models of biomolecular interaction networks. Genome Res. 2003;13:2498-504.

78. Blom J, Albaum SP, Doppmeier D, Puhler A, Vorholter FJ, Zakrzewski M, et al. EDGAR: a software framework for the comparative analysis of prokaryotic genomes. BMC Bioinformatics. 2009;10:154.

79. Battistuzzi FU, Feijao A, Hedges SB. A genomic timescale of prokaryote evolution: insights into the origin of methanogenesis, phototrophy, and the colonization of land. BMC Evol Biol. 2004;4:44.

80. Guindon S, Dufayard JF, Lefort V, Anisimova M, Hordijk W, Gascuel O. New Algorithms and Methods to Estimate Maximum-Likelihood Phylogenies: Assessing the Performance of PhyML 3.0. Syst Biol. 2010:59:307-21.

81. Hall BG. Building phylogenetic trees from molecular data with MEGA. Mol Biol Evol. 2013;30:1229-35.

82. Langille MG, Brinkman FS. IslandViewer: an integrated interface for computational identification and visualization of genomic islands. Bioinformatics. 2009;25:664-5.

83. Waack S, Keller O, Asper R, Brodag T, Damm C, Fricke WF, et al. Score-based prediction of genomic islands in prokaryotic genomes using hidden Markov models. BMC Bioinformatics. 2006;7:142.

84. Hsiao W, Wan I, Jones SJ, Brinkman FSL. IslandPath: aiding detection of genomic islands in prokaryotes. Bioinformatics (Oxford, England). 2003;19:418-20.

\section{Submit your next manuscript to BioMed Central and take full advantage of:}

- Convenient online submission

- Thorough peer review

- No space constraints or color figure charges

- Immediate publication on acceptance

- Inclusion in PubMed, CAS, Scopus and Google Scholar

- Research which is freely available for redistribution 